\title{
Novel short isoforms of adenylyl cyclase as negative regulators of cAMP production
}

\section{Authors:}

Benjamin Vallin ${ }^{1}$, Yohan Legueux-Cajgfinger ${ }^{1}$, Nathalie Clément ${ }^{1}$, Martine Glorian ${ }^{1}$, Laurent Duca $^{2}$, Pierre Vincent ${ }^{1, \dagger, *}$, Isabelle Limon ${ }^{1, \dagger, *}$ and Régis Blaise ${ }^{1, \dagger}$.

1: Sorbonne Université, Université Pierre et Marie Curie (UPMC), Institut de Biologie ParisSeine (IBPS), UMR CNRS 8256 Adaptation biologique et vieillissement (B2A), 75005 Paris, France.

2: UFR Sciences Exactes et Naturelles, Université de Reims Champagne Ardenne (URCA), UMR CNRS 7369 Matrice Extracellulaire et Dynamique Cellulaire (MEDyC), Laboratoire Signalisation et Récepteurs Matriciels (SiRMa), Campus Moulin de la Housse, 51687 Reims, France.

$\dagger$ : P.V, I.L and R.B equally contributed to this work.

*: to whom correspondence should be addressed: pierre.vincent@upmc.fr,

isabelle.limon@upmc.fr

Phone: +33 (0)144273716/2588

Fax: +33(0)144272650

Running title: Short adenylyl cyclases downregulate cAMP 


\begin{abstract}
Here, we cloned a new family of four adenylyl cyclase (AC) splice variants from transdifferentiated vascular smooth muscle cells (VSMCs) encoding short forms of AC8 that we have named "AC8E1-4" in accordance with the nomenclature of those previously identified. Using biosensor imaging and biochemical approaches, we showed that AC8E isoforms have no cyclase activity and act as dominant-negative regulators by forming heterodimers with other full-length ACs, impeding the traffic of functional units towards the plasma membrane. The existence of these dominant-negative isoforms may account for an additional unsuspected degree of cAMP signaling regulation. It also reconciles the induction of an AC in transdifferentiated VSMCs with the vasoprotective influence of cAMP. The generation of alternative splice variants of ACs may constitute a generalized strategy of adaptation to the cell's environment whose scope had so far been ignored in physiological and/or pathological contexts.
\end{abstract}

Keywords: adenylyl cyclase 8E / cAMP signaling / dominant-negative / heterodimerization / vascular smooth muscle cells. 


\section{Introduction}

Cells responses to their environment depend on the expression and membrane localization of adenylyl cyclases (ACs), which, together with cyclic nucleotide phosphodiesterases, are the principal enzymes controlling the cAMP signal. Nine membrane forms of adenylyl cyclases (ACs) have been identified in mammals, with discrete tissue distributions and unique regulatory properties, providing a potential focal point within the cell for the integration of diverse stimuli [1,2]. In vascular smooth muscle cells (VSMCs), it has been shown that i) changes in the AC population expressed in cells derived from hamster vas deferens smooth muscle modify the processing of stimulatory and inhibitory input [3], ii) differences between the $\mathrm{Ca}^{2+}$-sensitive $\mathrm{AC}$ isoforms of SMCs determine the effect of a vasopressin agonist on isoproterenol-stimulated cAMP [4], iii) the cAMP produced by AC isoform 6 (AC6) specifically enhances cAMP-mediated cytoskeletal reorganization without affecting cell growth due to its precise location within the membrane [5], iv) AC6 causes hyaluronan-mediated intimal thickening of the ductus aerius, whereas AC2 inhibits AC6induced hyaluronan production [6].

We have shown in vitro that the full transition of rat VSMCs towards a migratory/inflammatory phenotype (referred to as a transdifferentiation process) is dependent on the de novo expression of AC8 [7,8]. By contrast, in a non-inflammatory context, differentiated/contractile VSMCs mostly express AC isoforms 3, 5, and 6 [4,9]. In a mouse model of atherosclerosis, knocking out the AC8 gene decreases the inflammation of the abdominal aorta (Limon unpublished) and atherosclerotic lesions [10]. We have expanded these in vivo findings by showing, on human samples, that the neointimal VSMCs have high levels of AC8, whereas very few AC8-positive VSMCs are detected in the medial layer in either atherosclerotic or healthy arteries [11]. The causal relationship between AC8 expression and the migratory response of transdifferentiated VSMCs (tdVSMCs) is associated in the rat in vivo model of post-angioplasty restenosis with a marked transient upregulation of AC8 expression in VSMCs migrating to form the intimal cushion [11].

This increase in adenylyl cyclase expression was surprising. Indeed, increases in intracellular cAMP concentration $\left([\mathrm{cAMP}]_{\mathrm{i}}\right)$ have been shown to inhibit the migratory [12], proliferative [13] and inflammatory [14] properties of tdVSMCs. In addition, many studies have shown that increases in $[\mathrm{cAMP}]_{\mathrm{i}}$ in tdVSMCs antagonize pathological vascular remodeling [15-19]. 
Four different splice variants of AC8, encoding the AC8A-D isoforms, have been identified in human, mice and/or rat so far [20-23]. AC8A-D all display different structure, subcellular location and enzymatic activity [20,22].

We show here that the AC expressed de novo in tdVSMCs correspond to a family of short AC8 proteins derived from new AC8 splice variantsthe AC8 gene by alternative splicing, that we have named "E" in accordance with the nomenclature of previously identified variants [20,22]. AC8E isoforms are catalytically inactive and act as dominant-negatives by dimerizing with endogenous full-length adenylyl cyclases and retaining them in the rough endoplasmic reticulum (RER), thereby reducing global cAMP production within the cell. The existence of these dominant-negative isoforms may account for an additional, unsuspected degree of cAMP signaling regulation, and reconcile the de novo expression of an $\mathrm{AC}$ with the transdifferentiation process of VSMCs. 


\section{Materials and methods}

\subsection{Reagents}

Dulbecco's modified Eagle's medium (DMEM), type I collagen from calf skin, poly-L-lysine, L-glutamine, penicillin, streptomycin, thapsigargin and 3-isobutyl-1-methylxanthine (IBMX) were purchased from Sigma-Aldrich, Saint Quentin Fallavier, France. We obtained fetal calf serum (FCS) and collagenase from Gibco BRL, Cergy-Pontoise, France and G418 from InvivoGen, San Diego, CA, USA. Elastase and the LightCycler 480 SYBR Green I Master mix were obtained from Roche Diagnostics, Meylan, France. Recombinant human interleukin-1 $\beta$ (IL-1 $\beta$ ) was purchased from PeproTech, Neuilly-sur-Seine, France, and forskolin (fsk) was obtained from Tocris Bioscience, Bristol, UK. Opti-MEM, Lipofectamine RNAiMAX, M-MLV reverse transcriptase, oligo(dT) primers, RNaseOUT recombinant ribonuclease inhibitor,

NuPAGE LDS sample buffer and polyacrylamide gels were obtained from Invitrogen, Life Technologies, Saint-Aubin, France. We purchased the QIAquick gel extraction kit from Qiagen, Les Ulis, France. FuGENE HD, the ReliaPrep RNA Cell Miniprep System and GoTaq DNA polymerase were purchased from Promega, Charbonnières-les-Bains, France. Control and AC8 small interfering RNAs (siRNAs) were obtained from siTOOLs Biotech GmbH, Planegg, Germany. ArrayScript reverse transcriptase, Platinum SuperFi DNA polymerase, Halt protease inhibitor cocktail, EZ-Link Sulfo-NHS-SS-Biotin and streptavidin agarose resin were obtained from Thermo Fisher Scientific, Villebon sur Yvette, France. We purchased GelRed from Biotium, Fremont, CA, USA and oligonucleotides from Eurofins Genomics, Les Ulis, France. The DC protein assay and the Clarity western ECL substrate were purchased from Bio-Rad, Marnes-la-Coquette, France. Protein G PLUS-Agarose was obtained from Santa Cruz Biotechnology, Inc., Dallas, TX, USA and nitrocellulose membranes were purchased from Amersham, Courtaboeuf, France. N-glycosidase F was obtained from New England Biolabs, Évry, France. We obtained the cAMP - Gs HiRange kit from Cisbio Bioassays, Codolet, France and the Dako fluorescence mounting medium from Dako, Carpinteria, CA, USA.

\subsection{Cell culture}


Adult male Wistar rats (Janvier Labs, Le Genest-Saint-Isle, France) were ethically sacrificed by an intraperitoneal injection of pentobarbital $\left(300 \mathrm{mg} \cdot \mathrm{kg}^{-1}\right)$ and VSMCs were isolated from thoracic aortas as previously described [7], according to European Directive 2010/63/EU and the rules laid down by the university ethics committee. Primary VSMC cultures were subcultured from passages 1 to 3 in culture dishes coated with type I collagen from calf skin. Cells were grown at $37^{\circ} \mathrm{C}$, under an atmosphere containing $5 \% \mathrm{CO}_{2}$, in DMEM containing 1 g.L $\mathrm{L}^{-1}$ glucose, $4 \mathrm{mM}$ L-glutamine, $10 \% \mathrm{FCS}, 100 \mathrm{U} \cdot \mathrm{mL}^{-1}$ penicillin, and $100 \mu \mathrm{g} . \mathrm{mL}^{-1}$ streptomycin. VSMCs were starved by incubation for $12 \mathrm{~h}$ in serum-free DMEM before treatment with IL-1 $\beta\left(10 \mathrm{ng} \cdot \mathrm{mL}^{-1}\right)$ for $72 \mathrm{~h}$.

HEK-293 cells were grown at $37^{\circ} \mathrm{C}$, under an atmosphere containing $5 \% \mathrm{CO}_{2}$, in DMEM containing 4.5 g.L $\mathrm{L}^{-1}$ glucose, $4 \mathrm{mM}$ L-glutamine, 5\% FCS, $100 \mathrm{U}^{\mathrm{mL}} \mathrm{m}^{-1}$ penicillin, and 100 $\mu \mathrm{g} . \mathrm{mL}^{-1}$ streptomycin.

\subsection{Transfection}

Before transfection with siRNAs, VSMCs at $80 \%$ confluence were incubated for $2 \mathrm{~h}$ in antibiotic-free DMEM containing 1 g.L $\mathrm{L}^{-1}$ glucose, $4 \mathrm{mM}$ L-glutamine and 10\% FCS. Cells were then transfected by adding $0.25 \%$ Lipofectamine RNAiMAX and $5 \mathrm{nM}$ siRNAs diluted in $12.5 \%$ final Opti-MEM. The AC8 siRNA is a mixture of 30 different siRNAs directed against mRNA regions conserved in all AC8 splice variants (reference sequence: NM_017142.1). Cells were transferred, $24 \mathrm{~h}$ after transfection, to starvation (serum-free) medium for $12 \mathrm{~h}$.

Plasmid transfection was systematically performed with HEK-293 cells at $80 \%$ confluence in complete growth medium, in the presence of FuGENE HD and Opti-MEM, according to the information provided in the FuGENE HD Protocol Database (Promega). Cells were systematically used $48 \mathrm{~h}$ post-transfection. Stable transfection was performed in the same complete growth medium supplemented with $0.8 \mu \mathrm{g} \cdot \mathrm{mL}^{-1} \mathrm{G} 418$ antibiotic for one week, and monoclonal cell populations were generated by limiting dilution.

\subsection{Cloning strategies}

To clone the AC8 splice variants expressed in tdVSMCs, cells were treated with IL-1 $\beta$ (10 ng. $\mathrm{mL}^{-1}$ ) for $72 \mathrm{~h}$ and total RNA was extracted with the ReliaPrep RNA Cell Miniprep System. Total RNA $(1 \mu \mathrm{g})$ was then reverse-transcribed with ArrayScript reverse transcriptase 
and $2.5 \mu \mathrm{M}$ oligo(dT) primers. The cDNAs encoding the rat AC8 were amplified with Platinum SuperFi DNA polymerase, using phosphorylated forward and reverse primers (Table $\mathrm{S} 1$ ) binding to the 5 '-untranslated region and the 3'-untranslated region of the rat AC8A cDNA (NM_017142.1), respectively. The final PCR products were visualized by electrophoresis in a $1 \%$ agarose gel. Amplicons of the expected size were purified with the QIAquick gel extraction kit and blunt-cloned into the EcoRV site of pcDNA3. Positive clones were sequenced and four cDNAs encoding AC8 were identified and named AC8E1, E2, E3 and $\mathrm{E} 4$.

We obtained pcDNA3-VSV by inserting a double-stranded HindIII/KpnI-compatible end oligonucleotide adaptor (Table S1), including the vesicular stomatitis virus glycoprotein tag sequence (VSV), between the HindIII and KpnI restriction sites of pcDNA3. AC8A, AC8E1 and AC8E4 coding sequences were amplified by PCR with primers deleting the start codon (Table S1). The purified PCR fragments were inserted between the KpnI and XbaI restriction sites of pcDNA3-VSV to generate plasmids encoding N-terminally VSV-tagged AC8A, AC8E1 and AC8E4.

We generated the HA-AC3-expressing vector by inserting a double-stranded EcoRI/BamHIcompatible end oligonucleotide adaptor (Table S1), including the human influenza hemagglutinin (HA) epitope sequence, in frame with the first 26 coding bases of the rat AC3 sequence (from +3 to +29 , including the internal rAC3 BamHI site), in place of the EcoRI/BamHI fragment of pcDNA3-rAC3 (a generous gift from C. Dessauer).

All clones and constructs were sequenced on both strands, by dye-labeling chain-terminator chemistry methods, with an ABI 3730xl DNA Analyzer (Thermo Fisher Scientific, Villebon sur Yvette, France).

\subsection{PCR}

Total RNA was extracted with the ReliaPrep RNA Cell Miniprep System, according to the manufacturer's protocol. After annealing oligo $(\mathrm{dT})$ primers $(1 \mu \mathrm{M})$ to template RNAs $(0.5-1$ $\mu \mathrm{M}$ ) at $70^{\circ} \mathrm{C}$ for $5 \mathrm{~min}$, we initiated primer extension by adding M-MLV reverse transcriptase plus $0.5 \mathrm{mM}$ dNTPs, 1 unit of RNaseOUT recombinant ribonuclease inhibitor and $20 \mathrm{mM}$ DTT, and incubated the reaction mixture for a further $60 \mathrm{~min}$ at $37^{\circ} \mathrm{C}$.

Quantitative PCR was performed with a LightCycler 480 instrument as described previously [24]. The forward and reverse primers used for selective amplification of the cDNAs 
encoding rat cyclophilin A and ACs are presented in Table S1. Real-time quantitative PCR data are presented as the amount of each target mRNA relative to the amount of mRNAs for the cyclophilin A housekeeping reference gene.

For the relative quantification of the various AC8 transcripts, cDNAs from IL-1 $\beta$-treated VSMCs (equivalent to $50 \mathrm{ng}$ of total RNA) were amplified in a Prime Full Size Thermal Cycler (Techne, UK) with 1.25 U of GoTaq DNA polymerase, $1.5 \mathrm{mM} \mathrm{MgCl}_{2}, 0.2 \mathrm{mM}$ of each dNTP, and $0.5 \mu \mathrm{M}$ of forward and reverse primers. Primer pairs for RT-PCR analysis were tested for self-complementarity, dimer formation and melting temperature with Serial Cloner 2-6-1 software. PCR was performed with the following thermal settings: denaturation and enzyme activation at $95^{\circ} \mathrm{C}$ for $2 \mathrm{~min}$, with 25 to 33 cycles of $95^{\circ} \mathrm{C}$ for $1 \mathrm{~min}, 58$ to $64^{\circ} \mathrm{C}$ for $1 \mathrm{~min}$, and $72^{\circ} \mathrm{C}$ for $1 \mathrm{~min}$, followed by a final extension at $72^{\circ} \mathrm{C}$ for $5 \mathrm{~min}$. The sizes of the amplified fragments were determined by GelRed staining after electrophoresis in a $2 \%$ agarose gel. The PCR fragments were visualized with a E-BOX VX5 digital image processor (Vilber Lourmat, Collégien, France) and signal intensity was determined with Image J software. The forward and reverse primers used for selective amplification of the cDNAs encoding AC8 variants are presented in Table S1.

\subsection{Preparation of cell lysates}

HEK-293 cells were washed with ice-cold phosphate-buffered saline (PBS), scraped into RIPA lysis buffer (25 mM Tris-HCl, pH 7.5, $150 \mathrm{mM} \mathrm{NaCl,} \mathrm{1 \%} \mathrm{NP40,} \mathrm{1 \%} \mathrm{sodium}$ deoxycholate, $0.1 \%$ SDS, Halt protease inhibitor cocktail) and subjected to three five-second bursts of sonication with a Sonifier 150 (Branson Ultrasonics, Danbury, CT, USA). The resulting cell lysates were briefly incubated on a rotating wheel for $15 \mathrm{~min}$ at $4{ }^{\circ} \mathrm{C}$ and then cleared by centrifugation $\left(13,000 \times \mathrm{g}\right.$ for $15 \mathrm{~min}$ at $\left.4^{\circ} \mathrm{C}\right)$. Protein concentrations were determined with the DC protein assay. Proteins were then mixed with the NuPAGE LDS sample buffer and denatured for $1 \mathrm{~h}$ at $37^{\circ} \mathrm{C}$ before SDS-PAGE analysis.

When indicated, whole-cell lysates were treated with N-glycosidase F before electrophoresis. Briefly, proteins $(20 \mu \mathrm{g})$ were denatured by heating at $100^{\circ} \mathrm{C}$ for $10 \mathrm{~min}$ in $0.5 \% \mathrm{SDS}$ and 40 mM DTT. Denatured proteins were incubated with 500 units of N-glycosidase F or the same volume of $50 \%$ glycerol (controls) in $20 \mathrm{mM}$ Tris- $\mathrm{HCl} \mathrm{pH} 7.5,50 \mathrm{mM} \mathrm{NaCl}, 5 \mathrm{mM}$ EDTA and $1 \% \mathrm{NP}-40$ for $1 \mathrm{~h}$ at $37^{\circ} \mathrm{C}$. Proteins were then mixed with the NuPAGE LDS sample buffer and incubated for $5 \mathrm{~min}$ at $100^{\circ} \mathrm{C}$ before SDS-PAGE analysis. 


\subsection{Subcellular fractionation}

HEK-293 cells grown in 10-cm dishes were washed with ice-cold PBS and lysed by passing 10 times through a 26-gauge needle in fractionation (F) buffer containing $20 \mathrm{mM}$ HEPES pH 7.4, $250 \mathrm{mM}$ sucrose, $10 \mathrm{mM} \mathrm{KCl}, 1.5 \mathrm{mM} \mathrm{MgCl}_{2}, 1 \mathrm{mM}$ EDTA, $1 \mathrm{mM}$ EGTA, $1 \mathrm{mM}$ DTT and Halt protease inhibitor cocktail. The lysate was first centrifuged at $600 \mathrm{x} g$ for $10 \mathrm{~min}$. The supernatant (supernatant 1) was then centrifuged at $10,000 \mathrm{x} g$ for $15 \mathrm{~min}$ to isolate the heavy membrane pellet. The resulting supernatant 2 was centrifuged at $100,000 \times g$ for $1 \mathrm{~h}$ in an Optima MAX-XP ultracentrifuge (Beckman Coulter, Brea, CA, USA) to separate the light membrane pellet from the cytoplasmic fraction. The heavy and light membrane pellets were then resuspended in F buffer, passed through a 26-gauge needle 10 times and recentrifuged at the speeds indicated above. The resulting pellets, corresponding to the heavy membrane and light membrane fractions, were washed twice with PBS and resuspended in a final PBS buffer supplemented with $2 \%$ SDS.

\subsection{Cell surface biotinylation}

HEK-293 cells grown in 6-cm dishes were biotinylated in the presence of PBS+ $(0.1 \mathrm{mM}$ $\mathrm{CaCl}_{2}, 1 \mathrm{mM} \mathrm{MgCl}_{2}$, pH 8.0) supplemented with $1.5 \mathrm{mg} \cdot \mathrm{mL}^{-1}$ sulfosuccinimidyl-2(biotinamido) ethyl-1,3-dithiopropionate (EZ-Link Sulfo-NHS-SS-Biotin) for $45 \mathrm{~min}$ at $4^{\circ} \mathrm{C}$. Cells were then incubated for another $45 \mathrm{~min}$ at $4^{\circ} \mathrm{C}$ with a quenching solution containing PBS+ supplemented with $100 \mathrm{mM}$ glycine. Cells were washed with PBS and solubilized in lysis buffer $(20 \mathrm{mM}$ Tris $\mathrm{HCl} \mathrm{pH}$ 7.4, 2 mM EDTA, $2 \mathrm{mM}$ EGTA, 0.1\% SDS, 1\% Triton, Halt protease inhibitor cocktail). Biotinylated proteins were precipitated with streptavidin agarose resin suspended in $50 \mathrm{mM}$ Tris $\mathrm{HCl} \mathrm{pH} 7.4,100 \mathrm{mM} \mathrm{NaCl}, 50 \mathrm{mM}$ EDTA, Halt protease inhibitor cocktail. Precipitated proteins were resuspended in 2X NuPAGE LDS sample buffer, denatured for $30 \mathrm{~min}$ at $37^{\circ} \mathrm{C}$ and subjected to western blot.

\subsection{Co-immunoprecipitation}

HEK-293 cells grown in 10-cm dishes were washed with ice-cold PBS and lysed in $1 \mathrm{~mL}$ of solubilization (S) buffer (50 mM Tris pH 7.4, 1 mM EDTA, $150 \mathrm{mM} \mathrm{NaCl}, 0.3 \%$ (v/v) NP40, and Halt protease inhibitor cocktail). After incubation for $15 \mathrm{~min}$ on a rotating wheel, the cell suspension was passed 10 times through a 21-gauge needle and centrifuged at 10,000 x $g$ for 
$10 \mathrm{~min}$. The supernatant was then harvested, incubated on a rotating wheel for $1 \mathrm{~h}$ with $5 \mu \mathrm{l}$ of anti-HA or anti-VSV antibody (Table S2) and then incubated overnight with $40 \mu \mathrm{L}$ of $50 \%$ Protein G PLUS-Agarose bead slurry. Beads were washed five times in S buffer and complexes were eluted in $2 \mathrm{X}$ NuPAGE LDS sample buffer. The final eluate was vortexed vigorously and incubated for $1 \mathrm{~h}$ at $37^{\circ} \mathrm{C}$ before western blot analysis.

\subsection{Western blot}

Proteins were subjected to SDS-PAGE and transferred onto nitrocellulose membranes. Membranes were blocked by incubation for $1 \mathrm{~h}$ at room temperature with Tris-buffered saline supplemented with $0.1 \%$ Tween 20 and 5\% non-fat dried milk. Then, membranes were successively incubated with the primary antibodies indicated in Table S2 and horseradish peroxidase (HRP)-conjugated secondary antibodies. Signals were detected by enhanced chemiluminescence using the Clarity western ECL substrate, on Fujifilm LAS-300 (Fujifilm Medical Systems, Standford, CT, USA). When indicated, we used $\beta$-actin detection to control for equal protein loading and transfer efficiency.

\subsection{Immunocytochemistry}

HEK-293 cells were grown to subconfluence on poly-L-lysine-coated coverslips and fixed by incubation with 4\% PFA for $30 \mathrm{~min}$. PFA was then neutralized by incubation for $10 \mathrm{~min}$ with $50 \mathrm{mM} \mathrm{NH}_{4} \mathrm{Cl}$ at room temperature. Cells were then permeabilized by incubation with $0.2 \%$ Triton X-100 in PBS for 5 min. Before immunodetection, non-specific sites were blocked by incubating cells in PBS supplemented with 5\% donkey serum, $0.2 \%$ Triton X-100. Then, cells were successively incubated with primary antibodies directed against AC8 or the HA and VSV tags (Table S2) and Alexa Fluor conjugated secondary antibodies diluted in PBS supplemented with 5\% donkey serum. The coverslips were mounted in Dako fluorescence mounting medium. Images were acquired with a Leica SP5 confocal microscope (Leica Microsystems, Wetzlar, Germany).

\subsection{Measurement of cAMP dynamics}

Cells on collagen (VSMCs) or poly-L-lysine (HEK-293)-coated coverslips were infected with the ${ }^{\mathrm{T}} \mathrm{Epac}^{\mathrm{VV}}$-encoding adenoviral vector ( $\sim 100$ particles per VSMC) or transfected with the ${ }^{\mathrm{T}} \mathrm{Epac}^{\mathrm{VV}}$-encoding vector (HEK-293) for $24 \mathrm{~h}$. The generation of the ${ }^{\mathrm{T}} \mathrm{Epac}^{\mathrm{VV}}$-encoding adenovirus has been described elsewhere [24]. Coverslips were placed in a microscope 
chamber continually perfused $\left(2 \mathrm{~mL} \cdot \mathrm{min}^{-1}\right)$ with a buffer containing $125 \mathrm{mM} \mathrm{NaCl}, 2 \mathrm{mM}$ $\mathrm{CaCl}_{2}, 1 \mathrm{mM} \mathrm{MgCl} 2,1.25 \mathrm{mM} \mathrm{NaH} \mathrm{PO}_{4}, 26 \mathrm{mM} \mathrm{NaHCO}, 25 \mathrm{mM}$ glucose, maintained at $32^{\circ} \mathrm{C}$ and saturated with $95 \% \mathrm{O}_{2}-5 \% \mathrm{CO}_{2}$. Ratiometric analyses were performed as follows: fluorescence was excited with a LED source at $435 \mathrm{~nm}$, and fluorescence emission was monitored with a dichroic mirror (T450LPXR) and alternating emission filters for the donor (HQ480/40) and acceptor (D535/40). Pairs of images were recorded with a Orca-ER CCD camera (Hamamatsu Photonics, Japan), at 20-second intervals. Changes in $[\mathrm{cAMP}]_{\mathrm{i}}$ are expressed as the ratio of donor fluorescence (F480) to acceptor fluorescence (F535). The ratios were multiplied by a same constant for all experiments, such that the baseline ratio was 1 in basal conditions. The maximum ratio change ( $\operatorname{Rmax}$ ) was obtained by stimulating cells with $10 \mu \mathrm{M}$ fsk and $200 \mu \mathrm{M}$ IBMX. Filters and mirrors were obtained from AHF analysentechnik AG, Tübingen, Germany.

\subsection{CAMP accumulation assay}

cAMP accumulation in whole-cell lysates was measured with the cAMP - Gs HiRange kit after incubation for 60 minutes with the phosphodiesterase inhibitor IBMX (500 $\mu \mathrm{M})$ and the general AC activator forskolin $(10 \mu \mathrm{M})$, according to the manufacturer's instructions.

\subsection{Statistics}

All data are presented as the means \pm SEM of at least three independent experiments. The statistical significance of differences between groups was assessed with GraphPad Prism 6 (Graphpad Software Inc., La Jolla, CA, USA). The nonparametric two-tailed Mann-Whitney test was used for pairwise comparisons. The nonparametric two-tailed Kruskal-Wallis test and Dunn's post test were used for multiple comparisons. Differences were considered significant if $P<0.05$. 


\section{Results}

\subsection{The AC8 molecules expressed in transdifferentiated VSMCs belong to a novel}

family of AC8E isoforms, all lacking the first five transmembrane domains

We previously showed that the de novo expression of AC8 is a key feature of rat VSMCs undergoing transdifferentiation (tdVSMCs) in response to interleukin-1 $\beta$ (IL-1 $\beta$ ) $[7,8,11]$. Four different splice variants of AC8, encoding the AC8A-D isoforms, have been identified [20-23]. AC8A is the full-length isoform. AC8B, C and D variants lack exon 11, exon 8 and both these exons, respectively. We cloned the AC8 cDNAs from rat VSMCs stimulated with IL-1 $\beta$ (10 ng. $\left.\mathrm{mL}^{-1}\right)$ for 72 hours. All 13 clones sequenced displayed a non-frameshift $414 \mathrm{bp}$ deletion at the end of the exon 1, termed c.538_951del. They also displayed two other minor substitutions: a $\mathrm{CG}$ to GC missense mutation affecting bases 74 and 75 in exon 1 and resulting in the replacement of a glycine residue by an alanine residue in the protein, and a silent $\mathrm{G}$ to $\mathrm{C}$ mutation at base 3171 in exon 18 (Fig. 1A). The non-frameshift deletion stretches from a canonical alternative 5' donor site in exon 1 (E5SS, changing the 3' boundary of exon 1) to the $3^{\prime}$ acceptor splice site (3SS) of intron 1. Some cDNAs lack exon 8 and/or exon 11 (Fig. 1A and S1). Thus, tdVSMCs express a new family of four splice variants of the AC8 primary mRNA named AC8E1 to 4: the AC8E1 mRNA lacks only the end of exon 1, whereas the AC8E2, 3 and 4 mRNAs also lack exon 8, exon 11, and both these exons, respectively (Fig. 1A).

We then determined the relative abundances of AC8E1-4 transcripts and AC8A-D messengers (pooled together). We distinguished between these two pools of variants by amplifying cDNAs generated from tdVSMC mRNAs with forward (F) and reverse (R) primers annealing on either side of c.538_951del (Fig. 1B, left), assuming that the AC8E1-4 PCR products would be $395 \mathrm{bp}$ long whereas the AC8A-D PCR products would be $800 \mathrm{bp}$ long. We used cDNAs from contractile VSMCs and rat brain as negative and positive controls, respectively, for AC8 expression. As expected (Fig. 1B, middle) [25], an intense 800 bp band was detected in brain samples, in which the 395 bp band was barely visible; neither of these two bands was detected in contractile VSMC samples, confirming the lack of AC8 expression in these cells. In tdVSMC samples, the $395 \mathrm{bp}$ PCR products relating to AC8E1-4 were highly abundant, whereas the $800 \mathrm{bp}$ PCR products corresponding to AC8A-D were barely detectable. Based 
on a densitometric analysis of fluorescent amplicons, AC8Es accounted for more than $90 \%$ of total AC8 transcripts in tdVSMCs (Fig. 1B, right).

The amounts of the various AC8E mRNA subvariants were determined with forward (F) and reverse (R) primers binding to exons 7 and 12, respectively (Fig. 1C, left and middle). These amounts are expressed as a percent of total AC8E mRNAs: $15.5 \%$ for AC8E1, 18\% for AC8E2, 30.5\% for AC8E3 and 36\% for AC8E4 in tdVSMCs (Fig. 1C, right). All the AC8E transcripts lack the $414 \mathrm{bp}$ region encoding the putative first five transmembrane domains (TM), three extracellular and two intracellular loops of the enzyme (Fig. S1 and S2). The AC8E2 and AC8E3 mRNAs additionally lack either exon 8 (198 bp) encoding a 66-amino acid cytoplasmic domain corresponding to almost the entire $\mathrm{C} 1 \mathrm{~b}$ region, or exon 11 (90 bp) encoding a 30-amino acid extracellular domain corresponding to the TM9-10 loop, with two functional sites for N-linked glycosylation, respectively [26]. AC8E4 displays all three deletions. 
AC8A 1: ATGGAACTCTCGGATGTGCACTGCCTTAGCGGCAGCGAGGAACTCTATACCATTCACCCGACGCCCCCGGCCGCGGACGGCGGGA 85...518 GCCAGAGGCGC

AC8E1 1: ATGGAACTCTCGGATGTGCACTGCCTTAGCGGCAGCGAGGAACTCTATACCATTCACCCGACGCCCCCGGCGCCGGACGGCGGGA $85 . .518$ GCCAGAGGCGC

AC8E2 1: ATGGAACTCTCGGATGTGCACTGCCTTAGCGGCAGCGAGGAACTCTATACCATTCACCCGACGCCCCCGGCGCCGGACGGCGGGA 85...518 GCCAGAGGCGC

AC8E3 1: ATGGAACTCTCGGATGTGCACTGCCTTAGCGGCAGCGAGGAACTCTATACCATTCACCCGACGCCCCCGGCGCCGGACGGCGGGA 85...518 GCCAGAGGCGC

AC8E4 1: ATGGAACTCTCGGATGTGCACTGCCTTAGCGGAGCGAGGAACTCTATACCATTCACCCGACGCCCCCGGCGCCGGACGGCGGG 85 ...518 GCCAGAGGCGC

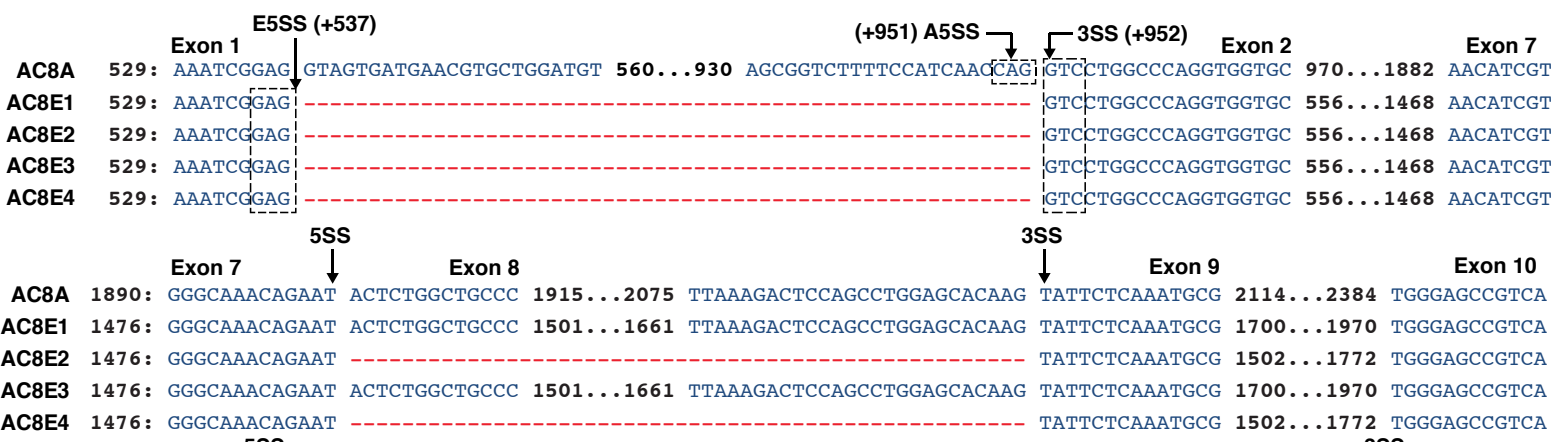
AC8E4 1476: GGGCAAACAGAAT -_-_-_-_._.-. TATTCTCAAATGCG 1502...1772 TGGGAGCCGTCA

$$
\text { Exon } 10 \downarrow
$$

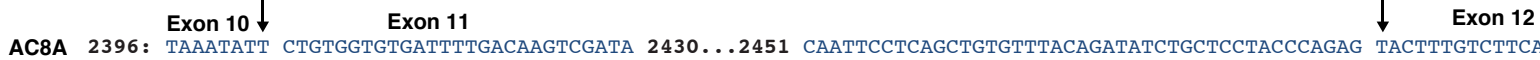
AC8E1 1982: TAAATATT CTGTGGTGTGATTTTGACAAGTCGATA 2016..2037 CAATTCCTCAGCTGTGTTTACAGATATCTGCTCCTACCCAGAG TACTTTGTCTTCA AC8E2 1784: TAAATATT CTGTGGTGTGATTTTGACAAGTCGATA 1818..1839 CAATTCCTCAGCTGTGTTTACAGATATCTGCTCCTACCCAGAG TACTTTGTCTTCA AC8E3 1982: TAAATATT -_-_-_- TACTTTGTCTTC

AC8E4 1784: TAAATATT TAСTTTGTCTTCA

AC8A 2507: CTGGGGTGTTGGCCATGGTGACGTGTGCAGT 2537_..3571 GCAGTTGTCCTTGGGCTTGTCCAG $3594 \ldots 3729$ CAAATCCGATTTGCCATAA : 3747

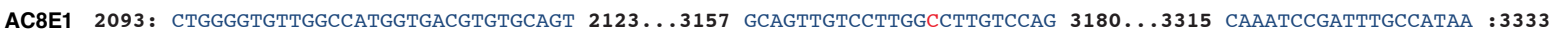
AC8E2 1895: CTGGGGTGTTGGCCATGGTGACGTGTGCAGT 1925_.2959 GCAGTTGTCCTTGGCCTTGTCCAG 2982...3117 CAAATCCGATTTGCCATAA :3135 AC8E3 2003: CTGGGGTGTTGGCCATGGTGACGTGTGCAGT 2033..3067 GCAGTTGTCCTTGGCCTTGTCCAG 3090...3225 CAAATCCGATTTGCCATAA : 3243 AC8E4 1805: CTGGGGTGTTGGCATGGTGACGTGTGCAGT 1835..2869 GCAGTTGTCCTTGGCCTTGTCCAG 2892 ...3027 CAAATCCGATTTGCCATAA :3045

B
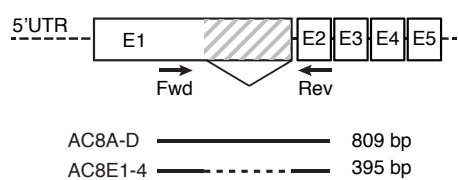

C

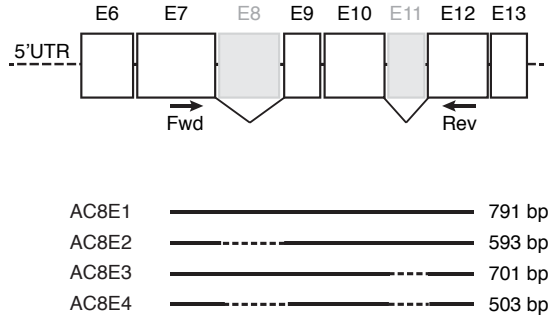

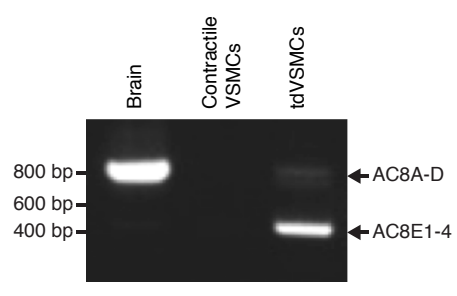

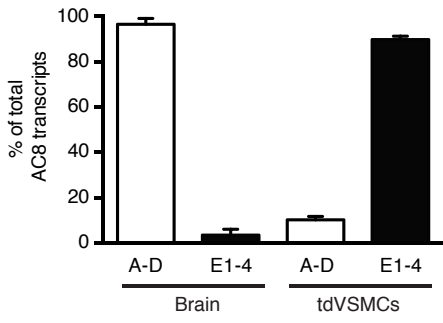

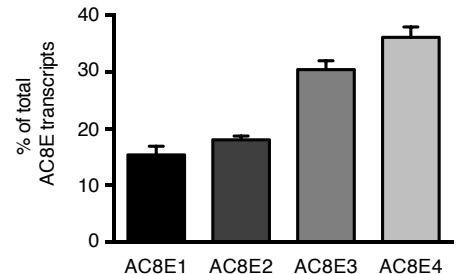

\section{Figure 1 - Four AC8 splice variants are expressed in tdVSMCs}

A Alignment of AC8A- and AC8E1-4 encoding sequences. Red dotted lines represent the nucleotide regions deleted in AC8E variants; black arrows indicate the 5' donor sites (5SS) and the 3' acceptor site (3SS) involved in alternative splicing; point mutations relative to the AC8A sequence are marked in red. 
B Abundance of AC8E transcripts relative to AC8A-D messengers. Left panel: diagram of the PCR strategy and resulting PCR products. Middle panel: agarose gel of the PCR products from rat brain, contractile (control) VSMC and tdVSMC cDNAs. Right panel: relative quantification of AC8A-D and AC8E1-4 mRNAs in brain and tdVSMCs, based on a densitometric analysis of fluorescent amplicons. Results are expressed in \% of total AC8 transcripts and represent the means \pm SEM of 4 independent experiments.

C Relative abundance of the AC8E1 to 4 variants in tdVSMCs. Left panel: diagram of the PCR strategy and resulting PCR products. Middle panel: agarose gel of the PCR products from tdVSMC cDNAs. Right panel: relative quantification of AC8E1-4 mRNAs in tdVSMCs. Results are expressed in \% of total AC8E transcripts and represent the means \pm SEM of 5 independent experiments.

\subsection{The de novo expression of AC8Es in transdifferentiated VSMCs downregulates cAMP production}

We then investigated a possible change in the dynamics of cAMP production associated with the de novo expression of AC8Es in VSMCs, using the FRET-based biosensor ${ }^{\mathrm{T}} \mathrm{Epac}^{\mathrm{VV}}$ [27]. Rat VSMCs were transdifferentiated by IL-1 $\beta$ treatment $\left(10 \mathrm{ng} \cdot \mathrm{mL}^{-1}\right)$ for 72 hours. Relative changes in $[\mathrm{cAMP}]_{\mathrm{i}}$ in response to the $\mathrm{AC}$ activator forskolin (fsk, $10 \mu \mathrm{M}$ ) were monitored over time, by calculating the ratio R of donor (F480 nm) to acceptor (F535 nm) fluorescence. cAMP level is reported as a percentage of the maximum ratio change (Rmax), corresponding to saturation of the biosensor. Rmax was systematically determined at the end of the experiment, by adding $200 \mu \mathrm{M}$ 3-isobutyl-1-methylxanthine (IBMX), a broad-spectrum phosphodiesterase inhibitor, in the presence of $10 \mu \mathrm{M}$ fsk. In contractile VSMCs, fsk addition increased the mean fluorescence ratio to $96.9 \pm 1.7 \%$ of the Rmax (Fig. 2A). By contrast, cAMP responses barely reached $53.3 \pm 6.6 \%$ of the Rmax in tdVSMCs. At the start of the experiment, the baseline ratio was similar for contractile VSMCs and tdVSMCs (Fig. S3A).

We used a siRNA strategy to investigate whether the smaller increase in $[\mathrm{cAMP}]_{i}$ in tdVSMCs was due to the de novo expression of AC8Es (Fig. 2B). As expected, in tdVSMCs transfected with the control siRNA, the response to forskolin was similar to that of untransfected tdVSMCs $(46.0 \pm 6.1$ vs. $53.3 \pm 6.6 \%$ of the Rmax, Fig. 2A vs. Fig. 2B). However, a partial recovery of the cAMP responses was observed in tdVSMCs transfected with the AC8 siRNA: in these cells, forskolin increased the fluorescence ratio to a 
significantly higher level than in tdVSMCs transfected with the control siRNA (65.5 $\pm 6.7 v s$. $46.0 \pm 6.1 \%$ of the Rmax, Fig. 2B). Baseline ratios were similar in tdVSMCs transfected with the control siRNA and in tdVSMCs transfected with the AC8 siRNA (Fig. S3B). The efficacy and specificity of the AC8 siRNA were demonstrated by real-time PCR $48 \mathrm{~h}$ post-transfection (Fig. S4). Together, these data demonstrate that the de novo expression of AC8Es profoundly affects cAMP dynamics by reducing the increase in $[\mathrm{cAMP}]_{\mathrm{i}}$ in tdVSMCs. This reconciles the increased expression of AC8 in atherosclerotic vessels with the reduced cAMP levels required for the pathological proliferation and migration of inflammatory VSMCs.

A

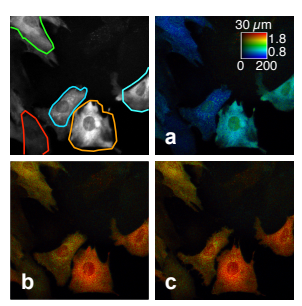

Contractile VSMCs

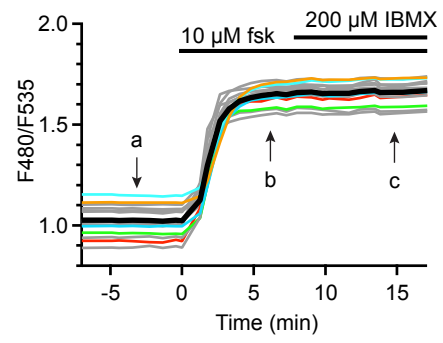

Transdifferentiated (td) VSMCs
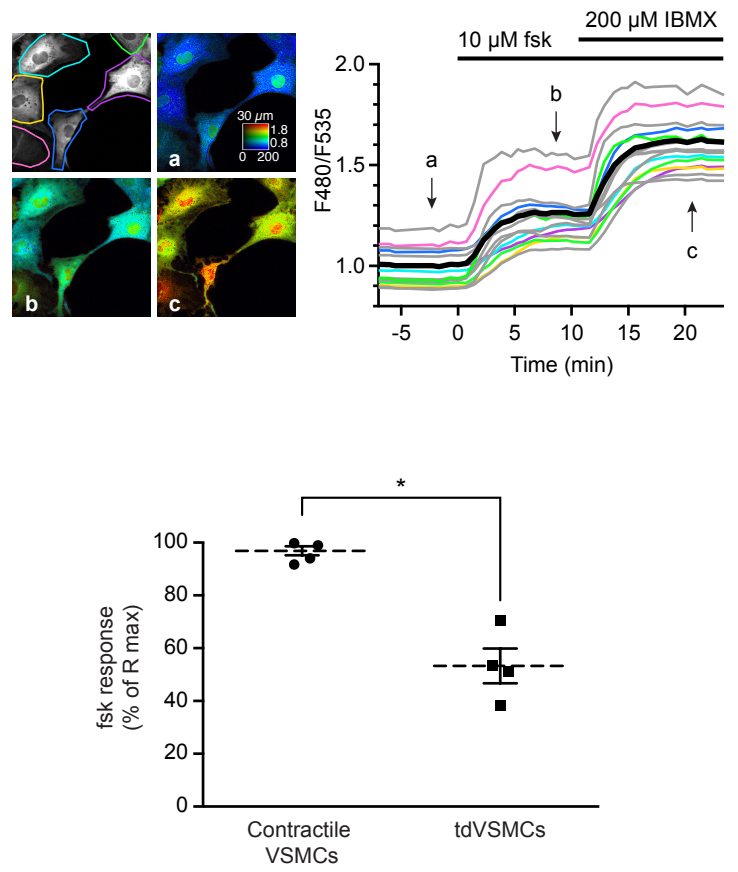

B
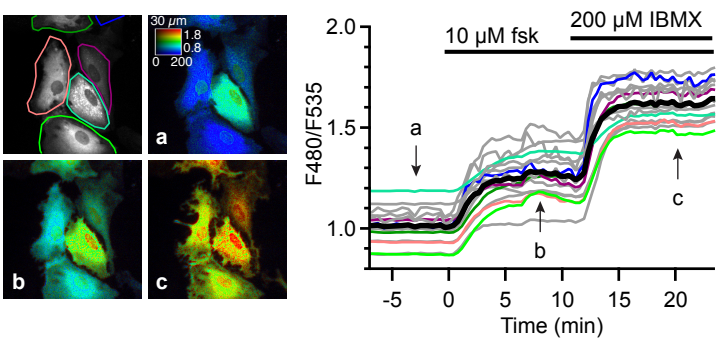

AC8 siRNA-transfected tdVSMCs
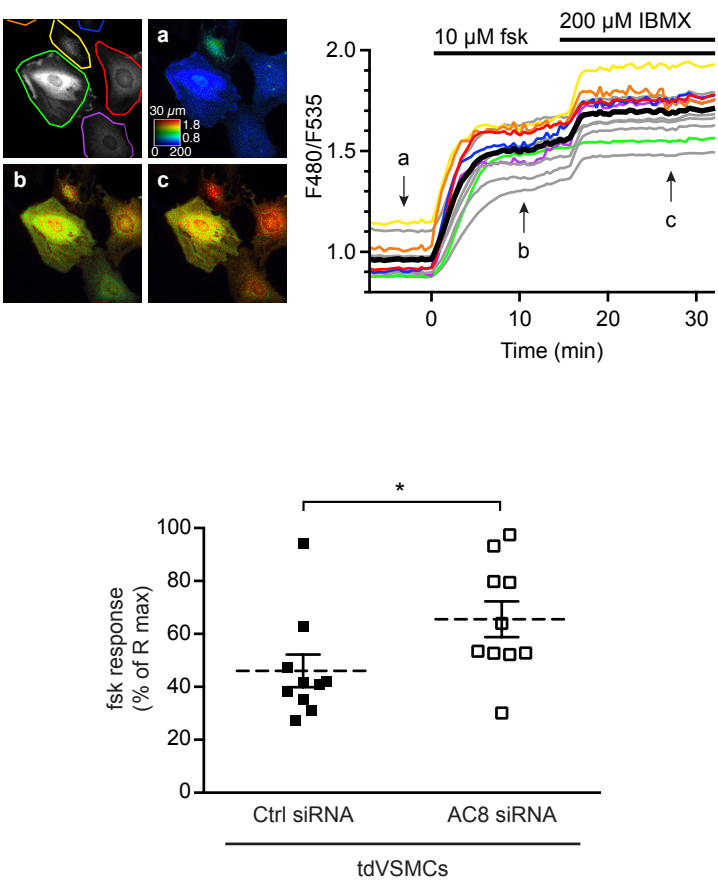
Figure 2 - The de novo expression of AC8Es in tdVSMCs reconfigures the dynamics of the cAMP signal

A, B Biosensor imaging of relative changes in $[\mathrm{cAMP}]_{\mathrm{i}}$ in transdifferentiated (td) versus contractile VSMCs (A) and in Ctrl siRNA-versus AC8 siRNA-transfected tdVSMCs (B). Left panels: microscopy fields in grayscale (top left) showing biosensor crude fluorescence at $535 \mathrm{~nm}$. The F480/F535 ratio was determined for individual cells, within regions of interest (ROI) delimited with colored contours. The calibration square indicates the range of intensity (in counts/pixel/s) horizontally and the F480/F535 ratio vertically. Pseudocolored images represent the F480/F535 ratio indicating the $[\mathrm{cAMP}]_{\mathrm{i}}$ (a) before treatment, (b) during $10 \mu \mathrm{M}$ fsk stimulation and (c) during the application of fsk $(10 \mu \mathrm{M})+\operatorname{IBMX}(200 \mu \mathrm{M})$. Right panels: each trace indicates the F480/F535 emission ratio over time in the ROI delimiting each cell. The black line represents the average of all traces. Gray traces correspond to the F480/F535 emission ratio of cells outside the displayed region. Lower panels: dot plot representing the mean values for fsk responses. Results are expressed as a percentage of the maximal ratio change (\% of the Rmax). Data shown are the means \pm SEM of at least $N=4$ independent experiments performed on $n=10$ to 20 individual cells. In (A), fsk responses were as follows: for contractile VSMCs: $96.9 \pm 1.7 \%, N=5$; for tdVSMCs: $53.3 \pm 6.6 \%, N=4, P=0.0159$. In (B), fsk responses were as follows: for Ctrl siRNA-transfected tdVSMCs: $46.0 \pm 6.1 \%, N=$ 10; for AC8 siRNA-transfected tdVSMCs: $65.5 \pm 6.7 \%, N=10, P=0.0288$. Side-by-side comparisons were performed with the Mann-Whitney test for unpaired data. *: $P<0.05$.

\subsection{Expression and localization of $\mathrm{AC8E}$ isoforms}

The lack of the TM9-10 loop with two functional sites for N-linked glycosylation in AC8B reduces its expression relative to that of AC8A and AC8C [20]. Since AC8E3 and 4 both miss this N-glycosylated extracellular domain, we next analyzed in western blot the protein expression of each AC8E isoform from four different clones of stably transfected HEK cells (Fig. 3A). The antibody we used was raised against the $\mathrm{C} 2 \mathrm{~b}$ terminal domain, a region that remains present in all $\mathrm{AC} 8 \mathrm{E}$ isoforms. $\mathrm{AC} 8 \mathrm{E} 1$ and $\mathrm{AC} 8 \mathrm{E} 2$ clones expressed high protein levels. Consistently with the study of Cali et al. [20], we found that AC8E3 and AC8E4 are very poorly expressed.

Subcellular fractionation experiments demonstrated that AC8E isoforms still insert into cellular membranes (Fig. 3B). Indeed, AC8E1-4 and the full-length AC8A mainly 
concentrated in the heavy membranes fraction and were absent from the cytosolic fraction. Heavy membranes include the plasma, endoplasmic reticulum and mitochondrial membranes, as attested by the levels of markers for these membranes, $\beta 1$-integrin, calnexin and cyclooxygenase IV (COX IV), respectively, and the absence of $\beta$-actin, a cytosolic marker. In AC8A HEK samples (Fig. 3B), the immunoreactive bands between the 180 and $250 \mathrm{kDa}$ molecular mass markers are of the size expected for AC8A monomers; the bands above 300 $\mathrm{kDa}$ are compatible with the predicted size of AC8A homodimers. In the samples derived from AC8E1-4 HEK, bands at or below $130 \mathrm{kDa}$ and those around $300 \mathrm{kDa}$ correspond to the monomeric and dimeric AC8E species, respectively (Fig. 3A and 3B). The anti-AC8 antibody does not cross-react with any endogenous $\mathrm{AC}$, as no bands were detected in the control HEK samples (Fig. 3B).

Immunocytochemistry showed AC8A at the plasma membrane whereas AC8E1-4 were intracellular and extranuclear (Fig. 3C). Hence, AC8A but not AC8E1 was detected when purifying biotinylated cell surface proteins (Fig. 3D). AC8E1 is unlikely targeted to mitochondria since it lacks the characteristic specific sequences (TargetP1.1, WoLF PSORT and Predotar 1.04 software). Consistent with AC8Es progressing through the RER, the migration profile of AC8E1 was efficiently altered by the exposure of cell lysates to $\mathrm{N}$ glycosidase F (Fig. S5). Interestingly, the migration patterns of AC8A and AC8E1 were differently sensitive to $\mathrm{N}$-glycosidase F: whereas AC8A showed a continuum of molecular species indicative of partial deglycosylation, the treatment had a less pronounced effect on AC8E1. Thus, AC8E1 likely possesses less diversified N-glycans motifs, in line with AC8E isoforms being, to some extent, stuck in the RER during the maturation process. In addition to demonstrating that AC8E1 and AC8E2 are the two most strongly expressed AC8E isoforms, these results indicate that all AC8Es are retained in the RER during the maturation process and form intermolecular dimers. 

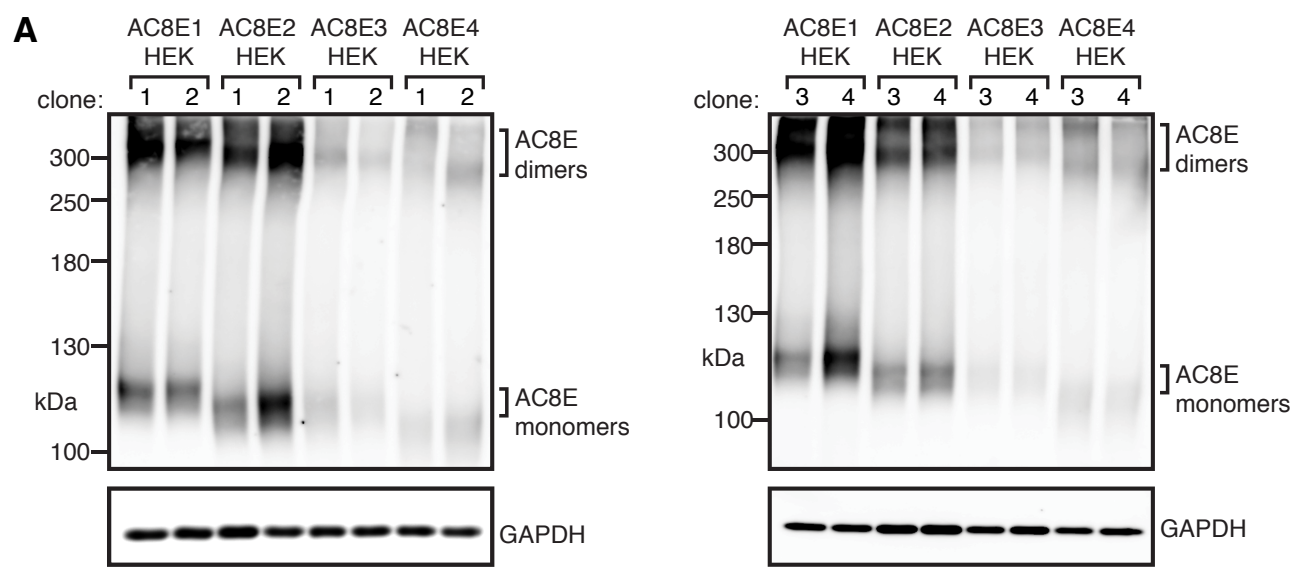

B

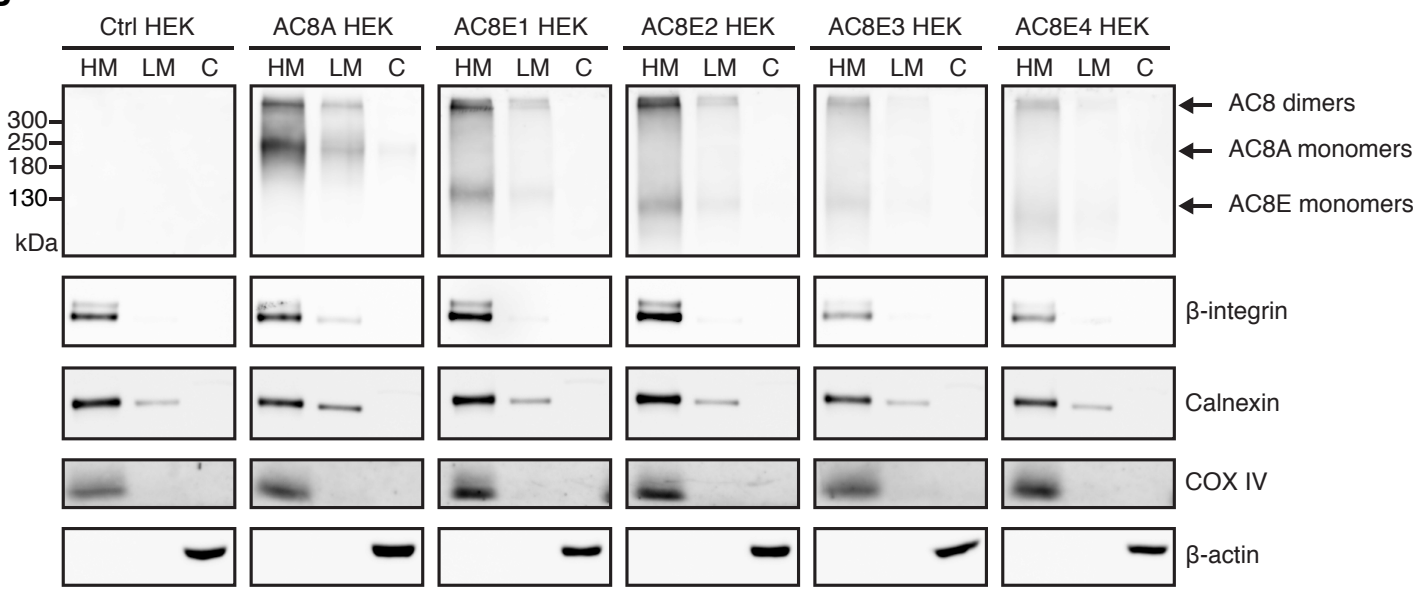

C

AC8A HEK

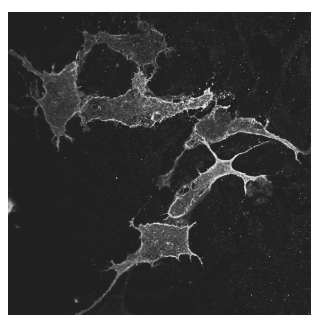

AC8E1 HEK

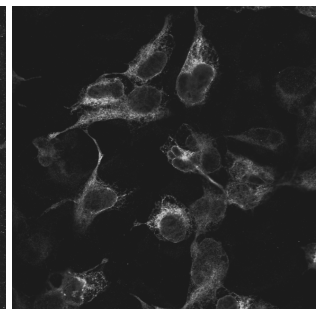

AC8E2 HEK

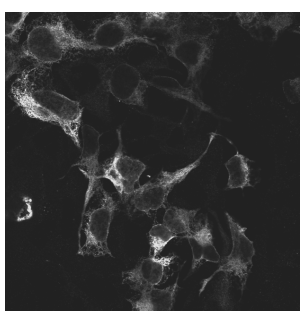

AC8E3 HEK

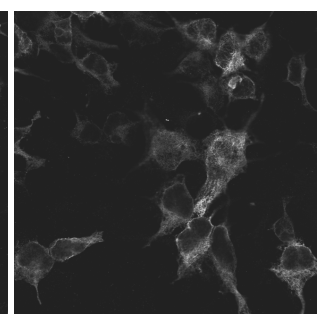

AC8E4 HEK

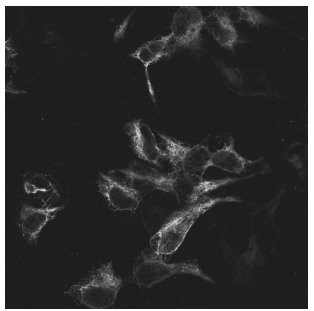

D
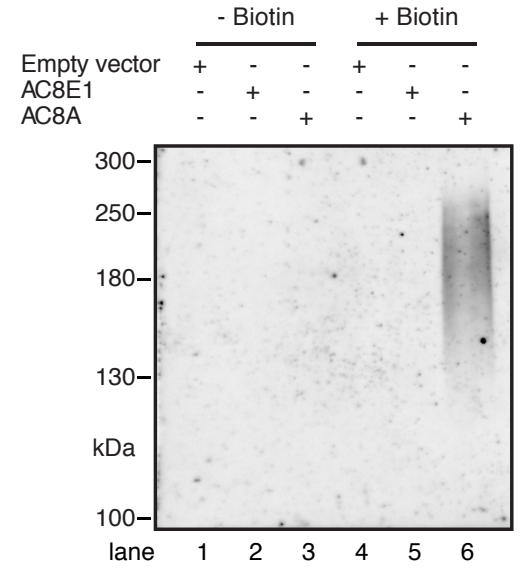

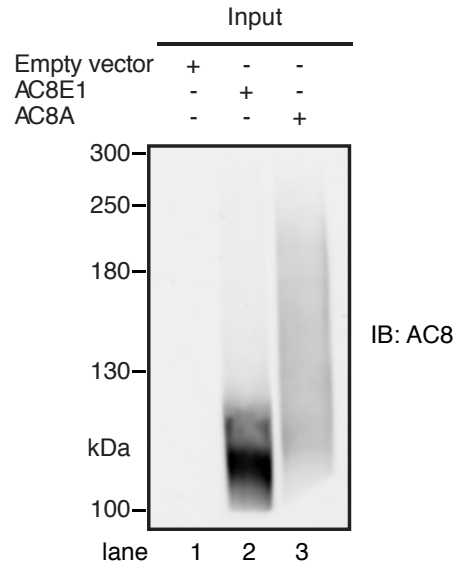




\section{Figure 3 - Expression and localization of AC8E isoforms}

A Western blot showing the stable expression of AC8E1, E2, E3 and E4 in HEK-293 cells. For each construct, four independent clones, numbered 1 to 4, were analyzed.

B Western blot showing the subcellular distribution of AC8A and AC8E1-4 in heavy membranes (HM), light membranes (LM) and the cytosol (C). $\beta$-integrin, calnexin, cyclooxygenase IV (COX IV), and $\beta$-actin are markers of the plasma, rough endoplasmic reticulum and mitochondrial membranes and the cytosol, respectively.

C Immunostaining showing the subcellular distribution of AC8A, AC8E1-4 in HEK-293 cells. Slides were analyzed with a Leica SP5 confocal microscope (63x).

D Western blot analysis of cell surface biotinylated proteins purified from HEK-293 cells transfected with the empty vector or with AC8E1- or AC8A-encoding plasmids and inputs.

Data information: In (A-D), the western blot and immunofluorescence images shown are representative of at least 3 independent experiments. All AC8 immunodetections were performed with the anti-AC8 (R20) antibody.

\subsection{The AC8E isoforms are catalytically dead and lower intracellular cAMP concentration}

Consistently, only the expression of AC8E1 and AC8E2 in HEK cells significantly limits the increase in $[\mathrm{cAMP}]_{\mathrm{i}}$ in response to forskolin as compared to mock-transfected cells (Fig. 4A). Indeed, the mean fluorescence ratio was $51.1 \pm 4.1 \%$ and $57.2 \pm 2.8 \%$ of the Rmax in AC8E1- and AC8E2-transfected cells, respectively, whereas in control HEK cells or in AC8E3- and AC8E4-expressing cells it reached $87.8 \pm 6.1 \%, 75.5 \pm 2.5 \%$ and $76.6 \pm 4.0 \%$ of the Rmax, respectively. In AC8A-expressing HEK cells, forskolin alone leads to biosensor saturation $(101.0 \pm 0.6 \%$ of the Rmax); the basal ratio in AC8A HEK was higher than that in control HEK and AC8Es-transfected cells, consistent with the overexpression of AC8A increasing basal and induced cAMP production.

As shown previously [28], AC8A is activated by capacitative calcium entry (CCE). We next passively depleted calcium stores with the SERCA inhibitor thapsigargin $(500 \mathrm{nM})$ in the absence of extracellular calcium (1 mM EGTA), and AC activity was jump-started with a low dose of forskolin $(10 \mathrm{nM})$. The addition of extracellular calcium $\left(2 \mathrm{mM} \mathrm{CaCl}_{2}\right)$ led to $\mathrm{CCE}$, which increased intracellular cAMP levels in AC8A- but not in AC8E1-expressing cells (Fig. 
4B). This additional experiment shows that AC8E1 does not exhibit the typical calciumactivated cyclase activity of AC8A. Overall, these results demonstrate that the c.538_951 deletion, despite lying outside the catalytic domains, kills the enzymatic activity of AC8Es while retaining the ability to lower cAMP responses in AC8E1-2. 
Figure 4 - The AC8E isoforms are inactive and prevent increases in cellular cAMP

A

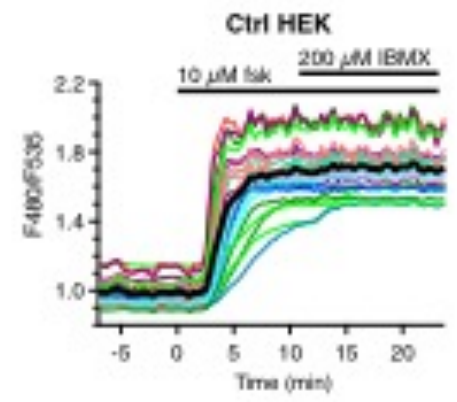

AC8E2 HEK

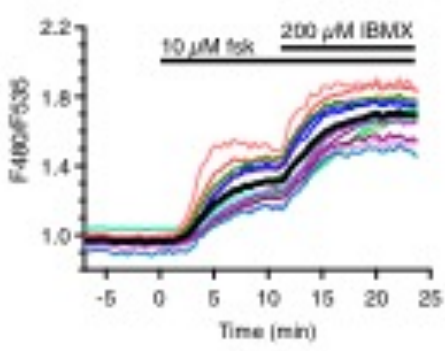

ACBA HEK

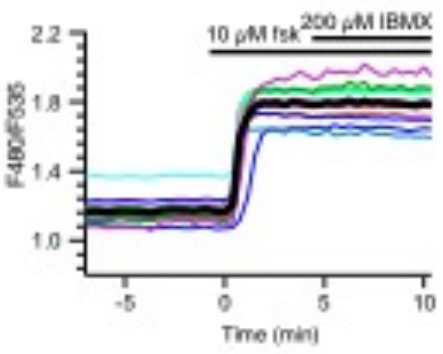

ACBE3 HEK

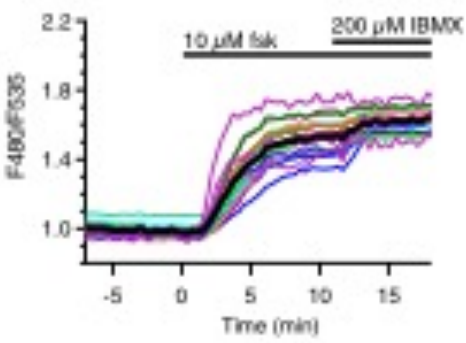

AC8E1 HEK

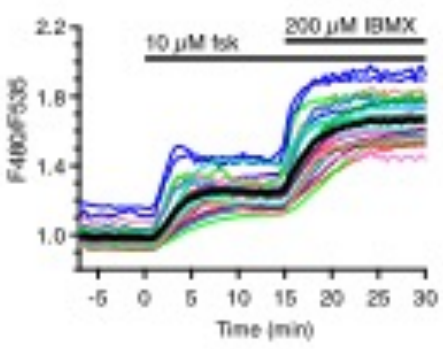

ACBEA HEK

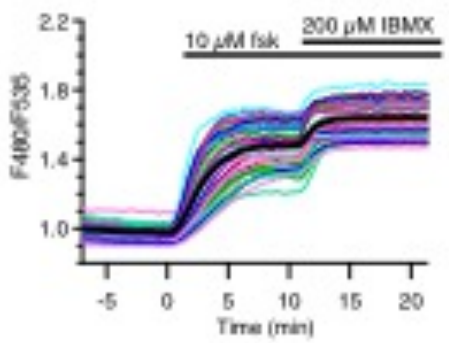

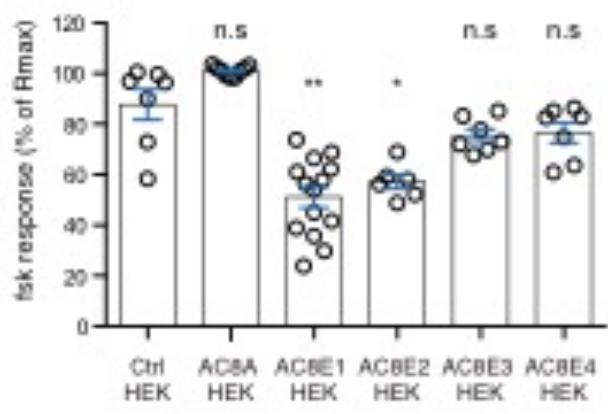

B

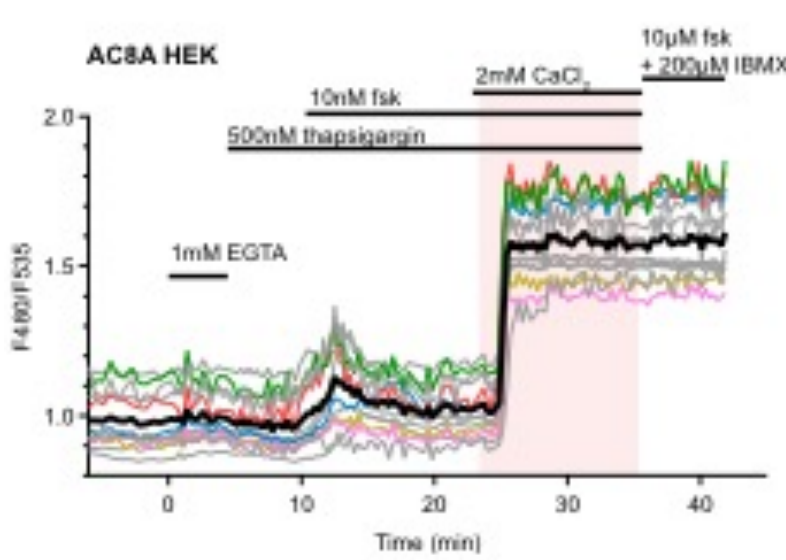

ACBE1 HEK

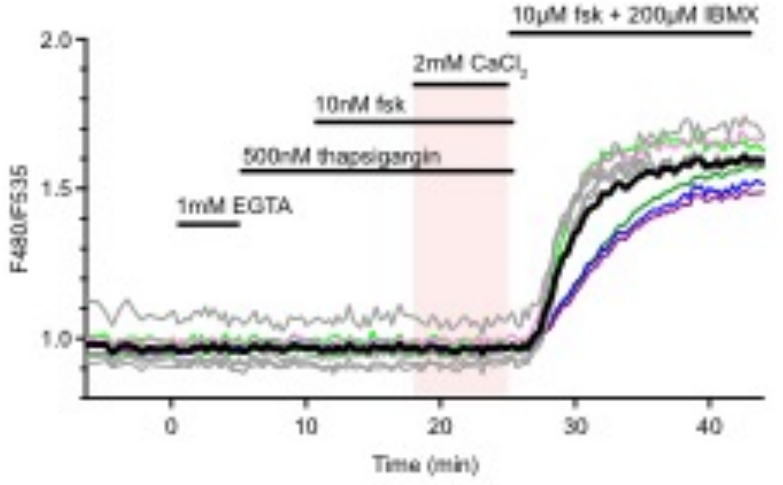


A Biosensor imaging of relative changes in $[\mathrm{cAMP}]_{\mathrm{i}}$ following treatment with forskolin (fsk, $10 \mu \mathrm{M}$ ), in control HEK clones and in clones stably expressing AC8A or AC8E1-4. The final application of fsk $(10 \mu \mathrm{M})$ together with IBMX $(200 \mu \mathrm{M})$ determined the maximal ratio change. Upper panels: each trace indicates the F480/F535 emission ratio over time in individual cells. The black line represents the average of all traces. Lower panel: dot plot and bars representing the mean values for fsk responses. Results are expressed as a percentage of the maximal ratio change ( $\%$ of the Rmax). Data shown are the means \pm SEM of at least $N=6$ independent experiments performed on independent clones. For each independent experiment, values represent the mean of $n=10$ to 20 individual cells. Fsk responses were as follows: for control HEK: $87.8 \pm 6.1 \%, N=7$; for AC8A HEK: $101.0 \pm 0.6 \%, N=9, P=0.6888$; for AC8E1 HEK: $51.1 \pm 4.1 \%, N=14, P=0.0030$; for AC8E2 HEK: $57.2 \pm 2.8 \%, N=6, P=$ 0.0366, for AC8E3 HEK: $75.5 \pm 2.5 \%, N=7, P>0.9999$; for AC8E4 HEK: $76.6 \pm 4.0 \%, N=$ 7, $P>0.9999$. Multiple comparisons to the control condition were performed with the Kruskal-Wallis test for unpaired data and Dunn's post test. **: $P<0.01,{ }^{*}: P<0.05$, ns: not significant.

B Biosensor imaging of relative changes in $[\mathrm{cAMP}]_{\mathrm{i}}$ in response to capacitive calcium entry in HEK-293 cells stably expressing AC8A or AC8E1. The final application of fsk (10 $\mu \mathrm{M})$ together with IBMX $(200 \mu \mathrm{M})$ determined the maximal ratio change. Each trace indicates the F480/F535 emission ratio over time in each cell. The black line represents the average of all traces. Data shown are representative of $N=3$ independent experiments performed on $n=10$ to 20 individual cells.

\subsection{The AC8E isoforms have dominant-negative effects on endogenous $\mathrm{AC}$ activity}

We hypothesized that AC8E expression reduced cAMP synthesis. We tested this hypothesis by stimulating ACs with a low dose of forskolin $(1 \mu \mathrm{M})$ while blocking phosphodiesterases with IBMX $(200 \mu \mathrm{M})$ to monitor the speed of cAMP synthesis with the FRET-based biosensor ${ }^{\mathrm{T}} \mathrm{Epac}^{\mathrm{VV}}$ (Fig. 5A). The ratio increase depends on the buffering effect of the biosensor on CAMP, and a slower onset is expected when the biosensor is present at a higher concentration. Therefore, for each cell, we plotted the slope at the origin of the ratio increase as a function of fluorescence intensity, used as a proxy for biosensor concentration. Regardless of fluorescence intensity, the initial slope of the ratio curve was shallower in AC8E1 HEK than in control HEK for a same amount of biosensor (Fig. 5B). In contrast and 
as expected, cells expressing AC8A showed a dramatically fast response onset. Similar results were obtained when performing cAMP accumulation assays in whole-cell lysates. AC8E1 significantly downregulated, by more than three times, whereas AC8A increased, by the same factor, the accumulation of cAMP after a 60-minute period of incubation with forskolin (10 $\mu \mathrm{M})$ plus IBMX $(500 \mu \mathrm{M})$ as compared to control HEK cells (Fig. 5C). Altogether, these data demonstrate that the inactive $\mathrm{AC} 8 \mathrm{E} 1$ isoform acts as a dominant-negative on endogenous $\mathrm{AC}$

A
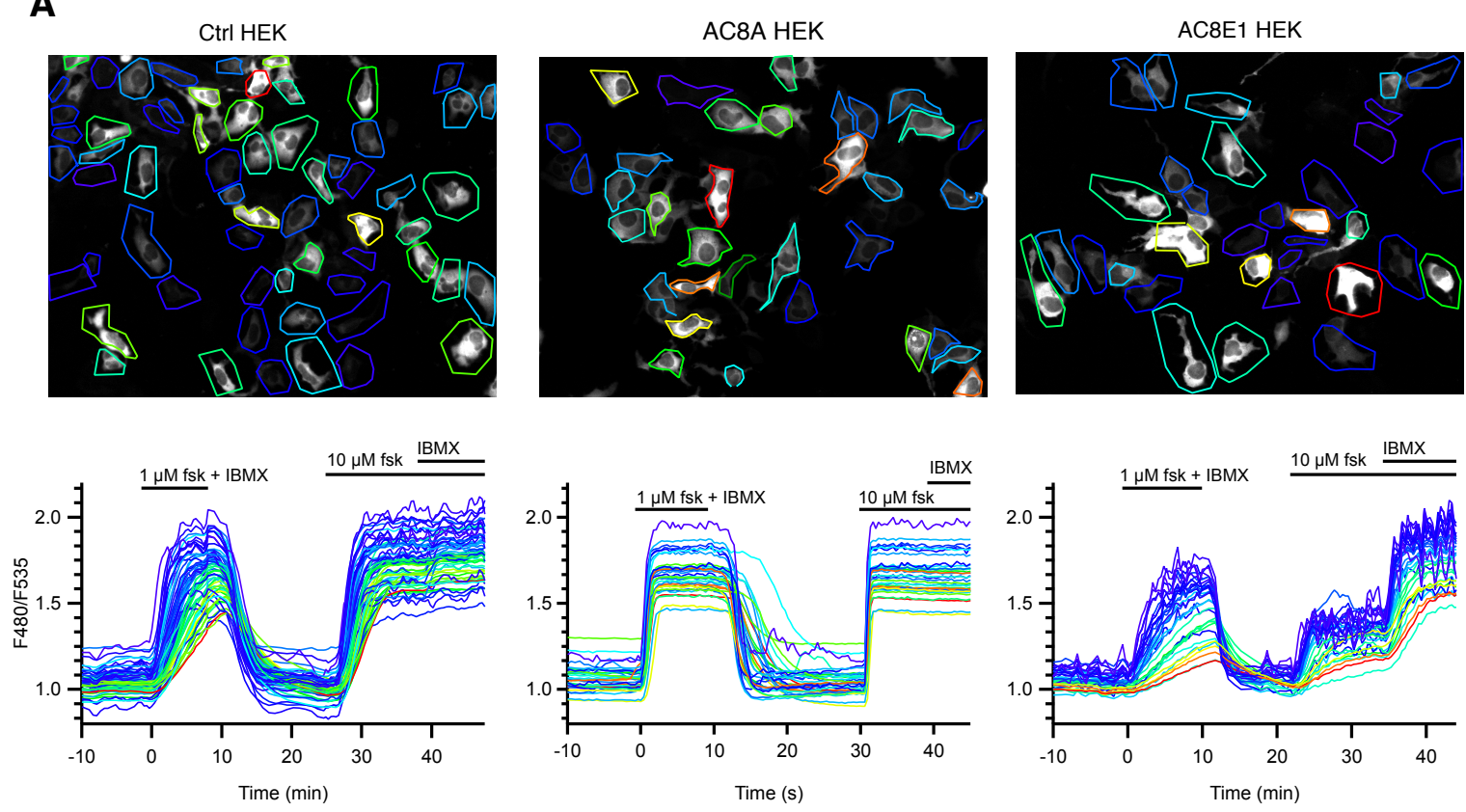

B

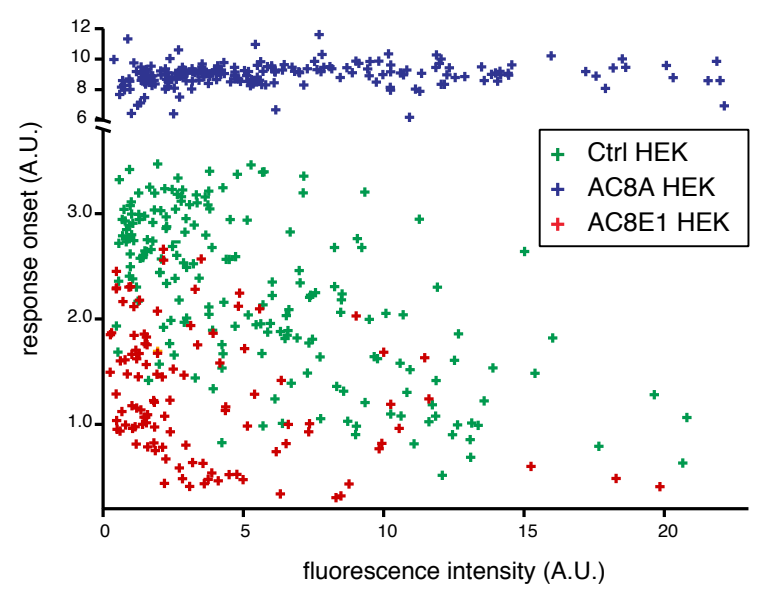

activity.
C

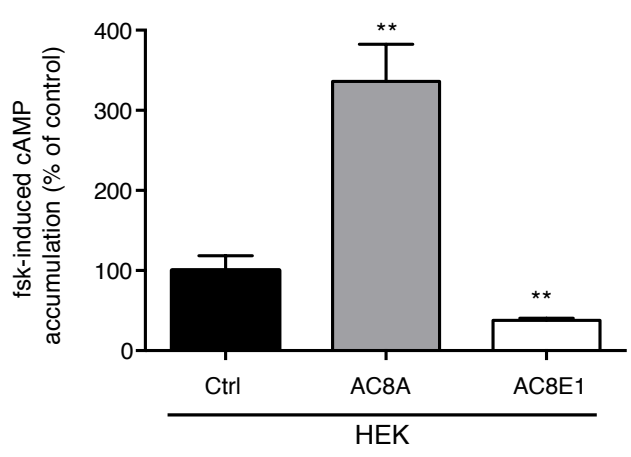




\section{Figure 5 - AC8E1 expression reduces cAMP production}

A Changes in $[\mathrm{cAMP}]_{\mathrm{i}}$ monitored with ${ }^{\mathrm{T}} \mathrm{Epac}^{\mathrm{VV}}$ in control (mock-transfected) HEK cells (left) or clones stably expressing AC8A (middle) or AC8E1 (right). A fast perfusion system was used to apply a low concentration of forskolin (fsk, $1 \mu \mathrm{M}$ ) to activate adenylyl cyclases moderately, in the presence of IBMX $(200 \mu \mathrm{M})$, to block cAMP degradation by phosphodiesterases. After recovery, the final application of fsk $(10 \mu \mathrm{M})$ together with IBMX $(200 \mu \mathrm{M})$ determined the maximal ratio change. Upper panels: for each cell, biosensor concentration was estimated by measuring the intensity of the brightest $10 \%$ of the pixels within the region of interest. The color code of each ROI reflects biosensor concentration: from blue (low) to red (high). Lower panels: traces indicating the F480/F535 emission ratio over time in the ROI delimiting each cell.

B Onset slope of the fluorescence ratio for each cell plotted against biosensor expression level. Results of 3 independent experiments are shown.

C cAMP accumulation assays on HEK cells transfected with empty vector (control), AC8A- or AC8E1-encoding vector. Results are expressed in \% of control and the means \pm SEM of 5 independent experiments are shown. Values were as follows: for AC8A HEK: $336.2 \pm 46.5 \%, P=0.0079$; for AC8E1 HEK: $37.8 \pm 2.6 \%, P=0.0079$. Side-by-side comparisons were performed with the Mann-Whitney test for unpaired data. $* *: P<0.01$.

\subsection{The dominant-negative effect of AC8E1 combines with direct AC interaction and the blocking of functional AC trafficking}

Previous studies have shown that heteromeric complexes assemble between full-length ACs and engineered deletion mutants [29,30]. We next explored whether the dominant-negative AC8E1 isoform directly influenced AC activity through heterodimer formation. We first assessed the ability of AC8E1 to interact with $\mathrm{AC} 3$, a major $\mathrm{AC}$ isoform expressed in contractile and transdifferentiated VSMCs [4]. HEK cells were transfected with VSV-tagged AC8E1 or HA-tagged full-length AC3 or both (Fig. 6A), and the interaction between these two proteins was assessed by co-immunoprecipitation. On western blots (Fig. 6B, upper left), after immunoprecipitation of the VSV-tagged AC8E1, we detected an immunoreactive band with the anti-HA antibody above $270 \mathrm{kDa}$ in samples from cells transfected with both the VSV - and HA-tagged constructs (lane 4). It corresponds to AC heterodimers containing glycosylated AC3 molecules. No bands were observed in the corresponding negative controls 
[cells transfected with either VSV-AC8E1 (lane 3) or HA-AC3 (lane 2) constructs or with the empty vector alone (lane 1)]. As expected, the anti-VSV antibody gave a similar pattern (Fig. 6B, lower left, lanes 3 and 4). Reverse co-immunoprecipitation with the anti-HA antibody for precipitation and the anti-VSV antibody for immunoblotting was just as effective (Fig. 6B, upper right).

This physical interaction translated into significantly lower levels of cAMP synthesis in response to forskolin in cells co-expressing AC3 and AC8E1 than in cells expressing AC3 only (Fig. 6C, 4 vs. 3). Again, the expression of AC8E1 alone decreased the forskolin-induced production of cAMP observed in pcDNA3 (control)-transfected cells (Fig. 6C, 2 vs. 1).

In addition, HA-AC3 was clearly present within the cell when co-expressed with VSVAC8E1, whereas it reached the plasma membrane when expressed with VSV-AC8A (Fig. 6D). The Pearson's colocalization coefficients with Costes'automatic thresholding (Fiji software Coloc2 plugin) were $r=0.86$ for AC8A/AC3 and $r=0.84$ for AC8E1/AC3.

Overall, these data demonstrate a dominant-negative effect of AC8E1 on AC3 function associated with a physical interaction between these two proteins, and they suggest that the AC8Es-dependent decrease in cAMP synthesis is due to a loss of AC trafficking to the plasma membrane. 
A
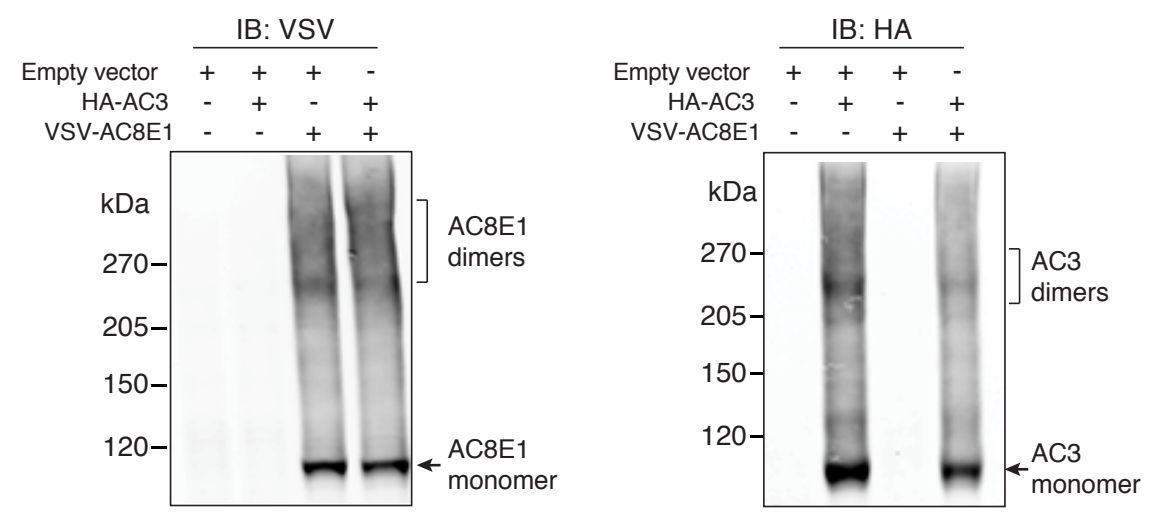

B
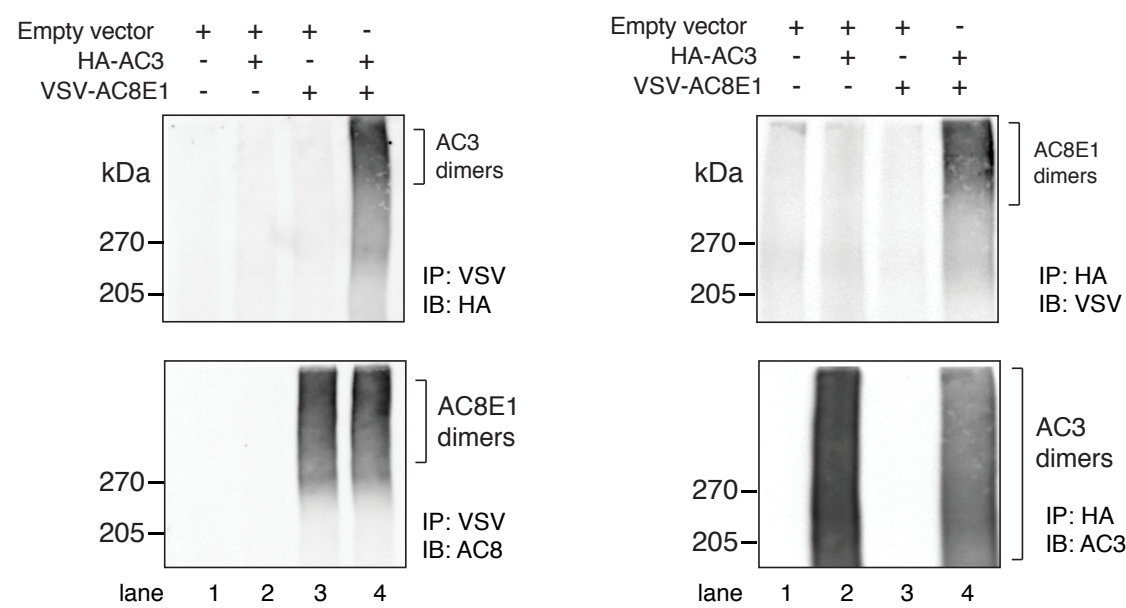

C
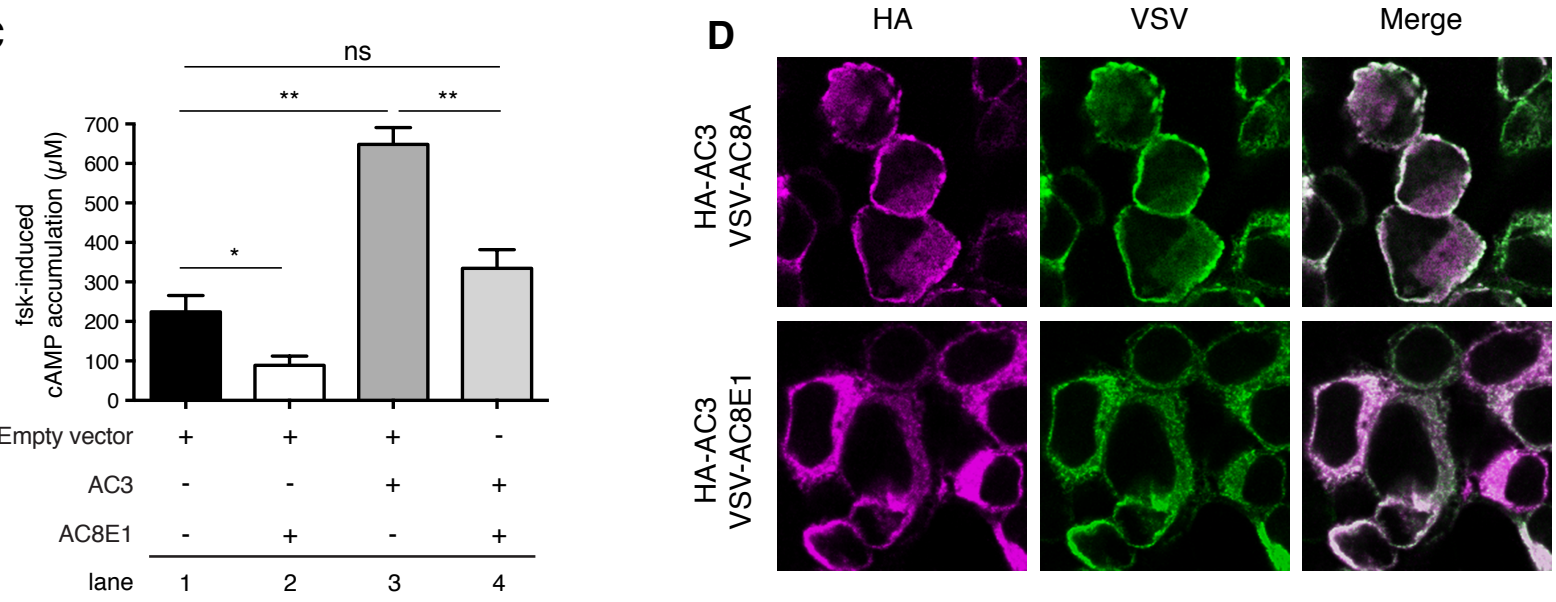

Figure 6 - AC8E1 heterodimerizes and inhibits AC activity

A Western blots showing AC8E1 (left panel) and AC3 (right panel) levels in HEK-293 cells transiently expressing VSV-AC8E1 and/or HA-AC3, probed with anti-VSV and anti-HA antibodies. 
B Western blots showing co-immunoprecipitation results. Immunoprecipitations (IPs) were performed with anti-VSV (left panel) and anti-HA (right panel) antibodies. Immunoblots (IBs) were performed with anti-AC8 or anti-VSV antibodies to detect AC8, and with antiAC3 or anti-HA antibodies to detect AC3.

C cAMP accumulation assays on HEK cells transfected with empty vector and/or AC8E1-encoding vector and/or or AC3-encoding vector, after $60 \mathrm{~min}$ of treatment with forskolin $(10 \mu \mathrm{M})$ plus IBMX $(500 \mu \mathrm{M})$. Results are expressed in $\mu \mathrm{M}$ and represent the means \pm SEM of 5 independent experiments. Values were as follows: for empty vectortransfected HEK: $224.4 \pm 41.3 \mu \mathrm{M}$; for AC8E1-expressing HEK: $88.9 \pm 23.2 \mu \mathrm{M}, P=$ 0.0159; for AC3-expressing HEK: $648.4 \pm 42.7 \mu \mathrm{M}, P=0.0079$; for AC8E1/AC3-expressing HEK: $334.5 \pm 47.5 \mu \mathrm{M}, P=0.0952$ when compared to control cells and $P=0.0079$ when compared to AC3-expressing cells. Side-by-side comparisons were performed with the nonparametric Mann-Whitney test for unpaired data. **: $P<0.01,{ }^{*}: P<0.05$, ns: not significant.

D Immunostaining of HEK-293 cells transfected with plasmids encoding HA-tagged AC3 and VSV-tagged AC8A or AC8E1. Confocal images were acquired with a Leica SP5 confocal microscope $(63 \mathrm{x})$.

Data information: In (A), (B) and (D), the western blot and immunofluorescence images shown are representative of at least 3 independent experiments. 


\section{Discussion}

The transdifferentiation process of medial VSMCs, which allows them to proliferate and migrate towards the intima, is a major cause of atherosclerosis and post-angioplasty restenosis. It has become clear that the contribution of VSMCs to atherosclerotic lesions has been greatly underestimated, because these cells may express macrophage, mesenchymal stem cell and/or myofibroblast markers in lesions [31]. Identification of the molecular mechanisms and entities driving changes in VSMC phenotype is therefore crucial for future drug development programs. We show here that VSMC transdifferentiation is associated with the de novo expression of a new family (the E family) of AC8 isoforms. These isoforms are catalytically inactive and have dominant-negative effects, decreasing total cAMP production. The inhibitory effect of AC8E1 on AC activity is accompanied by the formation of heterodimers between functional ACs and AC8E1. The de novo expression of members of the AC8E family may constitute a novel strategy of cellular desensitization to cAMP signaling distinct from those involving phosphodiesterases and multidrug resistance-associated proteins [32-35].

The lack of enzymatic activity of AC8E1 is consistent with the notion that the $\mathrm{C} 1 / \mathrm{C} 2$ interaction forming the catalytic site is not the only one interaction required for cyclase activity, instead being promoted by an intramolecular interaction between intact M1 and M2 transmembrane cassettes [36,37]. The need of this intramolecular interaction also accounts for improper transfer of AC8E isoforms towards the plasma membrane [36].

AC molecules within homo- and heterodimers of ACs cross-regulate each other. This is well illustrated by $i$ ) the work of Baragli et al., showing that AC2 and 5 assemble in vivo and exhibit enhanced cyclase activity as compared to AC2/AC2 or AC5/AC5 homodimers [38], ii) the study of Gu et al., demonstrating that the co-expression of a full-length AC8 together with an inactive engineered mutant (lacking part of the catalytic $\mathrm{C} 1$ domain) suppresses its activity [30]. Therefore, the negative regulation, by AC8E isoforms, of cAMP production by tdVSMCs is likely due to the ability of these isoforms to form complexes with endogenous full-length ACs. The findings of previous mutagenesis studies suggest that the region involved is the second transmembrane (M2) cassette [30,36]. The M2 hydrophobic cassette of AC8 contains seven SmxxxSm motifs (where Sm is a small residue: Gly, Ala, Ser or Thr) that have been proposed to drive TM helix dimerization in multi-pass proteins [39]. VSMCs 
mainly express AC3, 5 and 6 [4,9], all of which being capable of forming heterodimers with AC8 (Fig. 6B; [30]). Taking this supplementary information into account, we further propose that AC8Es inhibit cAMP synthesis within tdVSMCs by acting on AC isoforms 3, 5 and 6 .

Intact M1 and M2 cassettes within a single unit are essential for the correct translocation of AC8A to the plasma membrane (Fig. 3C and 3D; [36]). The overall decrease in cAMP production may, therefore, be due to AC8E isoforms retaining full length ACs in the RER. Several lines of evidence suggest that this is probably the case: i) AC3 was excluded from the plasma membrane when co-expressed with AC8E1 (Fig. 6D); ii) Ding and collaborators have demonstrated that a truncated form of AC6, similar to the AC8E isoforms in the sense that it also lacks one of the two membrane cassettes, prevents full-length AC6 molecules from reaching the plasma membrane [29]. The RER is the seat of protein quality control, addressing misfolded proteins to the ubiquitin-proteasome system for degradation. We therefore hypothesize that the lower levels of cAMP production observed in AC8E-expressing cells might be due to the degradation of AC dimers. In favor of that, the truncated form of AC6 mentioned by Ding and collaborators, that alters the trafficking of full-length AC molecules, also decreases their protein expression level. This would amount to a hijacking of the RER protein quality control mechanism from its original purpose, as it would prevent the trafficking of normal proteins to the plasma membrane. Together with alternative splicing, this process may constitute a new type of mechanism for regulating protein levels and/or signaling in cells. Although such a regulatory mechanism has never been described before, a role of alternative RNA processing in specifying the post-endocytic sorting of G-proteincoupled receptors has already been established [40].

Our finding that AC8E1 is N-glycosylated suggests that the two unique functional glycosylation sites of the TM9-10 loop are located within the RER lumen during protein processing (Fig. S5). According to the charge balance rule [41,42], the net charge difference of the 15 amino acids surrounding each end of a transmembrane domain determines its orientation in the lipid bilayer, with the most positive neighboring region facing the cytosol. It has also been shown that $i$ ) predictive transmembrane domains with a low hydrophobicity may become extramembrane domains and ii) highly hydrophobic transmembrane domains stabilize neighboring transmembrane domains of low hydrophobicity [43]. From the charge balance rule, the glycosylation status and the hydrophobic profile of AC8E1, one can assume that the remaining sixth transmembrane domain of the M1 cassette relocates to the cytosol 
and that the M2 cassette anchors to the RER membrane in the same orientation as AC8A. This is even more likely foreseen due to the fact that VSV-tagged AC8E1 molecules pulled-down endogenous AKAP79 in co-immunoprecipitation experiments, a cytosolic protein known to interact with the N-terminus of AC8A (Fig. S6). Based on that, and consistently with the study of Gu et al. [30], the whole N-terminus/TM6/C1 region of AC8E isoforms may be close enough $(<5 \mathrm{~nm})$ to inhibit their AC partners whether by direct interaction or by causing steric hindrance.

Finally, this discovery of novel AC splice variants specifically expressed in the pathological context of vascular remodeling is relevant to various diseases related to splicing defects, including cancers and neurological diseases [44]. The generation of AC alternative splice variants may therefore be part of a more general strategy of cell adaptation to its environment, the scope of which was previously unknown. Consistent with this, Katsushika and collaborators have described an AC5-alpha subvariant in dog heart encoding the first half of the enzyme only [45]. This variant is of particular interest because the heterodimerization motifs of the AC6 isoform, which belongs to the same family as AC5, appeared to be located in the M1 cassette [29]. Evidence for a functional impact of splice variants is accumulating $[46,47]$, but the causal contribution of disease-associated splice variants to the disease remains unclear in most cases. The functional assessment of AC5 and 8 splice variants in the heart and vessels could pave the way for further studies of the splice variants specifically expressed in a pathological context. 


\section{Acknowledgments}

We would like to thank S. Bolte and JF. Gilles from the imaging facility of the Paris-Seine Biological Institute (IBPS), C. Dessauer for providing the AC3 construct and JB. Klarenbeek for providing the ${ }^{\mathrm{T}} \mathrm{Epac}^{\mathrm{VV}}$ expression plasmid.

This work was supported by ANR grant 11BSV103401, the Lefoulon-Delalande Foundation, the French Federation of Cardiology (F.F.C), the Nouvelle Société Francophone d'Athérosclérose (NSFA), the Société Française de Cardiologie (SFC) and the Groupe de Réflexion sur la Recherche Cardiovasculaire (GRRC).

\section{Author contributions}

R.B and I.L came up with the initial concept for this study. B.V, Y.L-C, and R.B performed the key experiments. N.C constructed the ${ }^{\mathrm{T}} \mathrm{Epac}^{\mathrm{VV}}$-encoding adenovirus. M.G performed some of the PCR experiments. B.V, P.V, IL and R.B designed the study and the experiments and B.V, L.D, P.V, R.B and I.L interpreted the data. B.V, R.B, PV and I.L wrote the manuscript. Experiments were performed in the laboratories of P.V and I.L.

\section{Conflict of interest}

None declared. 


\section{References}

[1] N. Defer, M. Best-Belpomme, J. Hanoune, Tissue specificity and physiological relevance of various isoforms of adenylyl cyclase, Am. J. Physiol. Renal Physiol. 279 (2000) F400-416.

[2] R.K. Sunahara, C.W. Dessauer, A.G. Gilman, Complexity and diversity of mammalian adenylyl cyclases, Annu. Rev. Pharmacol. Toxicol. 36 (1996) 461-480. doi:10.1146/annurev.pa.36.040196.002333.

[3] A. Marjamaki, M. Sato, R. Bouet-Alard, Q. Yang, I. Limon-Boulez, C. Legrand, S.M. Lanier, Factors determining the specificity of signal transduction by guanine nucleotidebinding protein-coupled receptors. Integration of stimulatory and inhibitory input to the effector adenylyl cyclase, J. Biol. Chem. 272 (1997) 16466-16473.

[4] J.G. Webb, P.W. Yates, Q. Yang, Y.V. Mukhin, S.M. Lanier, Adenylyl cyclase isoforms and signal integration in models of vascular smooth muscle cells, Am. J. Physiol. Heart Circ. Physiol. 281 (2001) H1545-1552.

[5] R. Gros, Q. Ding, J. Chorazyczewski, J.G. Pickering, L.E. Limbird, R.D. Feldman, Adenylyl cyclase isoform-selective regulation of vascular smooth muscle proliferation and cytoskeletal reorganization, Circ. Res. $99 \quad$ (2006) 845-852. doi:10.1161/01.RES.0000245189.21703.c0.

[6] U. Yokoyama, S. Minamisawa, A. Katayama, T. Tang, S. Suzuki, K. Iwatsubo, S. Iwasaki, R. Kurotani, S. Okumura, M. Sato, S. Yokota, H.K. Hammond, Y. Ishikawa, Differential regulation of vascular tone and remodeling via stimulation of type 2 and type 6 adenylyl cyclases in the ductus arteriosus, Circ. Res. 106 (2010) 1882-1892. doi:10.1161/CIRCRESAHA.109.214924.

[7] N. Clément, M. Glorian, M. Raymondjean, M. Andréani, I. Limon, PGE2 amplifies the effects of IL-1beta on vascular smooth muscle cell de-differentiation: a consequence of the versatility of PGE2 receptors 3 due to the emerging expression of adenylyl cyclase $8, \mathrm{~J}$. Cell. Physiol. 208 (2006) 495-505. doi:10.1002/jcp.20673.

[8] Z. Keuylian, J.H.F. de Baaij, M. Gueguen, M. Glorian, C. Rouxel, E. Merlet, L. Lipskaia, R. Blaise, V. Mateo, I. Limon, The Notch pathway attenuates interleukin $1 \beta$ (IL1 $\beta$ )mediated induction of adenylyl cyclase 8 (AC8) expression during vascular smooth muscle cell (VSMC) trans-differentiation, J. Biol. Chem. $287 \quad$ (2012) 24978-24989. 
doi:10.1074/jbc.M111.292516.

[9] R.S. Ostrom, X. Liu, B.P. Head, C. Gregorian, T.M. Seasholtz, P.A. Insel, Localization of adenylyl cyclase isoforms and $\mathrm{G}$ protein-coupled receptors in vascular smooth muscle cells: expression in caveolin-rich and noncaveolin domains, Mol. Pharmacol. 62 (2002) 983-992.

[10] M. Gueguen, B. Vallin, M. Glorian, R. Blaise, I. Limon, Adénylyl cyclases et transdifférenciation des cellules musculaires lisses vasculaires: rôle dans le remodelage vasculaire pathologique, Biol. Aujourdhui. 210 (2016) 153-166. doi:10.1051/jbio/2016020.

[11] M. Gueguen, Z. Keuylian, V. Mateo, N. Mougenot, A.-M. Lompré, J.-B. Michel, O. Meilhac, L. Lipskaia, I. Limon, Implication of adenylyl cyclase 8 in pathological smooth muscle cell migration occurring in rat and human vascular remodelling, J. Pathol. 221 (2010) 331-342. doi:10.1002/path.2716.

[12] J.S. McKean, F. Murray, G. Gibson, D.A. Shewan, S.J. Tucker, G.F. Nixon, The cAMP-producing agonist beraprost inhibits human vascular smooth muscle cell migration via exchange protein directly activated by cAMP, Cardiovasc. Res. 107 (2015) 546-555. doi:10.1093/cvr/cvv176.

[13] R.C. Hewer, G.B. Sala-Newby, Y.-J. Wu, A.C. Newby, M. Bond, PKA and Epac synergistically inhibit smooth muscle cell proliferation, J. Mol. Cell. Cardiol. 50 (2011) 8798. doi:10.1016/j.yjmcc.2010.10.010.

[14] A. Chatterjee, A. Sharma, M. Chen, R. Toy, G. Mottola, M.S. Conte, The proresolving lipid mediator maresin 1 (MaR1) attenuates inflammatory signaling pathways in vascular smooth muscle and endothelial cells, PloS One. 9 (2014) e113480. doi:10.1371/journal.pone.0113480.

[15] Y. Cai, D.J. Nagel, Q. Zhou, K.D. Cygnar, H. Zhao, F. Li, X. Pi, P.A. Knight, C. Yan, Role of cAMP-phosphodiesterase 1C signaling in regulating growth factor receptor stability, vascular smooth muscle cell growth, migration, and neointimal hyperplasia, Circ. Res. 116 (2015) 1120-1132. doi:10.1161/CIRCRESAHA.116.304408.

[16] J.S. Douglas, D.R. Holmes, D.J. Kereiakes, C.L. Grines, E. Block, Z.M.B. Ghazzal, D.C. Morris, H. Liberman, K. Parker, C. Jurkovitz, N. Murrah, J. Foster, P. Hyde, G.B.J. Mancini, W.S. Weintraub, Cilostazol for Restenosis Trial (CREST) Investigators, Coronary stent restenosis in patients treated with cilostazol, Circulation. 112 (2005) 2826-2832. doi:10.1161/CIRCULATIONAHA.104.530097. 
[17] C. Indolfi, E. Di Lorenzo, A. Rapacciuolo, A.M. Stingone, E. Stabile, A. Leccia, D. Torella, R. Caputo, F. Ciardiello, G. Tortora, M. Chiariello, 8-chloro-cAMP inhibits smooth muscle cell proliferation in vitro and neointima formation induced by balloon injury in vivo, J. Am. Coll. Cardiol. 36 (2000) 288-293.

[18] Y. Inoue, K. Toga, T. Sudo, K. Tachibana, S. Tochizawa, Y. Kimura, Y. Yoshida, H. Hidaka, Suppression of arterial intimal hyperplasia by cilostamide, a cyclic nucleotide phosphodiesterase 3 inhibitor, in a rat balloon double-injury model, Br. J. Pharmacol. 130 (2000) 231-241. doi:10.1038/sj.bjp.0703287.

[19] N. Katakami, Y.-S. Kim, R. Kawamori, Y. Yamasaki, The phosphodiesterase inhibitor cilostazol induces regression of carotid atherosclerosis in subjects with type 2 diabetes mellitus: principal results of the Diabetic Atherosclerosis Prevention by Cilostazol (DAPC) study: a randomized trial, Circulation. $121 \quad$ (2010) 2584-2591. doi:10.1161/CIRCULATIONAHA.109.892414.

[20] J.J. Cali, R.S. Parekh, J. Krupinski, Splice variants of type VIII adenylyl cyclase. Differences in glycosylation and regulation by Ca2+/calmodulin, J. Biol. Chem. 271 (1996) 1089-1095.

[21] N. Defer, O. Marinx, D. Stengel, A. Danisova, V. Iourgenko, I. Matsuoka, D. Caput, J. Hanoune, Molecular cloning of the human type VIII adenylyl cyclase, FEBS Lett. 351 (1994) $109-113$.

[22] D. Delmeire, D. Flamez, S.A. Hinke, J.J. Cali, D. Pipeleers, F. Schuit, Type VIII adenylyl cyclase in rat beta cells: coincidence signal detector/generator for glucose and GLP1, Diabetologia. 46 (2003) 1383-1393. doi:10.1007/s00125-003-1203-8.

[23] L.M. Muglia, M.L. Schaefer, S.K. Vogt, G. Gurtner, A. Imamura, L.J. Muglia, The 5'flanking region of the mouse adenylyl cyclase type VIII gene imparts tissue-specific expression in transgenic mice, J. Neurosci. Off. J. Soc. Neurosci. 19 (1999) 2051-2058.

[24] K. Blirando, R. Blaise, N. Gorodnaya, C. Rouxel, O. Meilhac, P. Vincent, I. Limon, The stellate vascular smooth muscle cell phenotype is induced by IL-1 $\beta$ via the secretion of PGE2 and subsequent cAMP-dependent protein kinase A activation, Biochim. Biophys. Acta. 1853 (2015) 3235-3247. doi:10.1016/j.bbamcr.2015.09.019.

[25] A.C. Conti, J.W. Maas, L.M. Muglia, B.A. Dave, S.K. Vogt, T.T. Tran, E.J. Rayhel, L.J. Muglia, Distinct regional and subcellular localization of adenylyl cyclases type 1 and 8 in mouse brain, Neuroscience. 146 (2007) 713-729. doi:10.1016/j.neuroscience.2007.01.045. 
[26] M. Pagano, M.A. Clynes, N. Masada, A. Ciruela, L.-J. Ayling, S. Wachten, D.M.F. Cooper, Insights into the residence in lipid rafts of adenylyl cyclase AC8 and its regulation by capacitative calcium entry, Am. J. Physiol. Cell Physiol. 296 (2009) C607-619. doi:10.1152/ajpcell.00488.2008.

[27] J.B. Klarenbeek, J. Goedhart, M.A. Hink, T.W.J. Gadella, K. Jalink, A mTurquoisebased cAMP sensor for both FLIM and ratiometric read-out has improved dynamic range, PloS One. 6 (2011) e19170. doi:10.1371/journal.pone.0019170.

[28] A.C.L. Martin, D. Willoughby, A. Ciruela, L.-J. Ayling, M. Pagano, S. Wachten, A. Tengholm, D.M.F. Cooper, Capacitative $\mathrm{Ca} 2+$ entry via Orai1 and stromal interacting molecule 1 (STIM1) regulates adenylyl cyclase type 8, Mol. Pharmacol. 75 (2009) 830-842. doi:10.1124/mol.108.051748.

[29] Q. Ding, R. Gros, J. Chorazyczewski, S.S.G. Ferguson, R.D. Feldman, Isoformspecific regulation of adenylyl cyclase function by disruption of membrane trafficking, Mol. Pharmacol. 67 (2005) 564-571. doi:10.1124/mol.104.006817.

[30] C. Gu, J.J. Cali, D.M.F. Cooper, Dimerization of mammalian adenylate cyclases, Eur. J. Biochem. 269 (2002) 413-421.

[31] L.S. Shankman, D. Gomez, O.A. Cherepanova, M. Salmon, G.F. Alencar, R.M. Haskins, P. Swiatlowska, A.A.C. Newman, E.S. Greene, A.C. Straub, B. Isakson, G.J. Randolph, G.K. Owens, KLF4-dependent phenotypic modulation of smooth muscle cells has a key role in atherosclerotic plaque pathogenesis, Nat. Med. 21 (2015) 628-637. doi:10.1038/nm.3866.

[32] P.K. Bautista Niño, M. Durik, A.H.J. Danser, R. de Vries, U.M. Musterd-Bhaggoe, M.E. Meima, M. Kavousi, M. Ghanbari, J.H. Hoeijmakers, C.J. O’Donnell, N. Franceschini, G.M.J. Janssen, J.G.R. De Mey, Y. Liu, C.M. Shanahan, O.H. Franco, A. Dehghan, A.J.M. Roks, Phosphodiesterase 1 regulation is a key mechanism in vascular aging, Clin. Sci. Lond. Engl. 1979. 129 (2015) 1061-1075. doi:10.1042/CS20140753.

[33] S.D. Rybalkin, I. Rybalkina, J.A. Beavo, K.E. Bornfeldt, Cyclic nucleotide phosphodiesterase $1 \mathrm{C}$ promotes human arterial smooth muscle cell proliferation, Circ. Res. 90 (2002) 151-157.

[34] Y. Sassi, L. Lipskaia, G. Vandecasteele, V.O. Nikolaev, S.N. Hatem, F. Cohen Aubart, F.G. Russel, N. Mougenot, C. Vrignaud, P. Lechat, A.-M. Lompré, J.-S. Hulot, Multidrug resistance-associated protein 4 regulates cAMP-dependent signaling pathways and 
controls human and rat SMC proliferation, J. Clin. Invest. 118 (2008) 2747-2757. doi:10.1172/JCI35067.

[35] D.G. Tilley, D.H. Maurice, Vascular smooth muscle cell phenotype-dependent phosphodiesterase 4D short form expression: role of differential histone acetylation on cAMP-regulated function, Mol. Pharmacol. 68 (2005) 596-605. doi:10.1124/mol.105.014126.

[36] C. Gu, A. Sorkin, D.M. Cooper, Persistent interactions between the two transmembrane clusters dictate the targeting and functional assembly of adenylyl cyclase, Curr. Biol. CB. 11 (2001) 185-190.

[37] T. Seebacher, J.U. Linder, J.E. Schultz, An isoform-specific interaction of the membrane anchors affects mammalian adenylyl cyclase type V activity, Eur. J. Biochem. 268 (2001) 105-110.

[38] A. Baragli, M.-L. Grieco, P. Trieu, L.R. Villeneuve, T.E. Hébert, Heterodimers of adenylyl cyclases 2 and 5 show enhanced functional responses in the presence of Galpha s, Cell. Signal. 20 (2008) 480-492. doi:10.1016/j.cellsig.2007.10.033.

[39] A. Senes, M. Gerstein, D.M. Engelman, Statistical analysis of amino acid patterns in transmembrane helices: the GxxxG motif occurs frequently and in association with betabranched residues at neighboring positions, J. Mol. Biol. 296 (2000) 921-936. doi:10.1006/jmbi.1999.3488.

[40] M. Tanowitz, J.N. Hislop, M. von Zastrow, Alternative splicing determines the postendocytic sorting fate of G-protein-coupled receptors, J. Biol. Chem. 283 (2008) 35614 35621. doi:10.1074/jbc.M806588200.

[41] E. Hartmann, T.A. Rapoport, H.F. Lodish, Predicting the orientation of eukaryotic membrane-spanning proteins, Proc. Natl. Acad. Sci. U. S. A. 86 (1989) 5786-5790.

[42] M. Sato, R. Hresko, M. Mueckler, Testing the charge difference hypothesis for the assembly of a eucaryotic multispanning membrane protein, J. Biol. Chem. 273 (1998) 2520325208.

[43] M. Bogdanov, W. Dowhan, H. Vitrac, Lipids and topological rules governing membrane protein assembly, Biochim. Biophys. Acta. 1843 (2014) 1475-1488. doi:10.1016/j.bbamcr.2013.12.007.

[44] B. Chabot, L. Shkreta, Defective control of pre-messenger RNA splicing in human disease, J. Cell Biol. 212 (2016) 13-27. doi:10.1083/jcb.201510032.

[45] S. Katsushika, J. Kawabe, C.J. Homcy, Y. Ishikawa, In vivo generation of an 
adenylylcyclase isoform with a half-molecule motif, J. Biol. Chem. 268 (1993) 2273-2276.

[46] O. Kelemen, P. Convertini, Z. Zhang, Y. Wen, M. Shen, M. Falaleeva, S. Stamm, Function of alternative splicing, Gene. 514 (2013) 1-30. doi:10.1016/j.gene.2012.07.083.

[47] V. Pagliarini, C. Naro, C. Sette, Splicing Regulation: A Molecular Device to Enhance Cancer Cell Adaptation, BioMed Res. Int. 2015 (2015) 543067. doi:10.1155/2015/543067. 


\section{Supplementary figures}

AC8E1

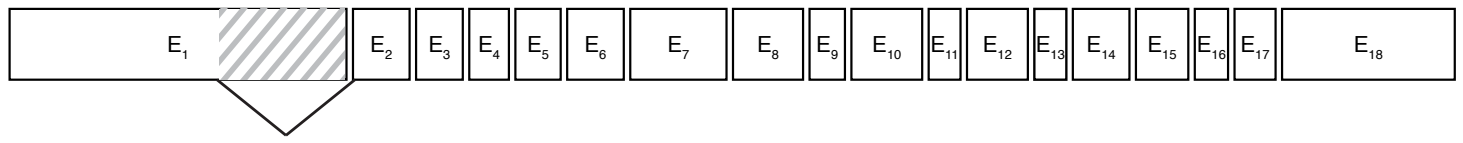

AC8E2

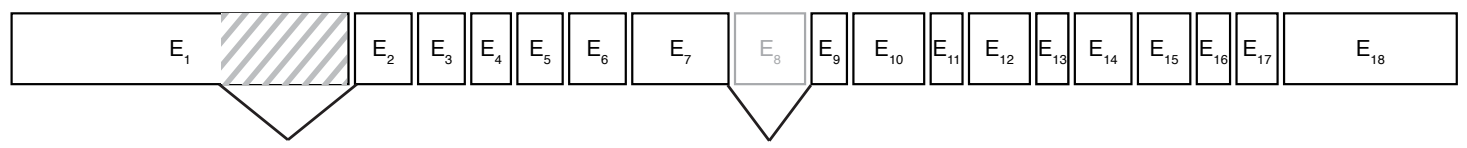

AC8E3

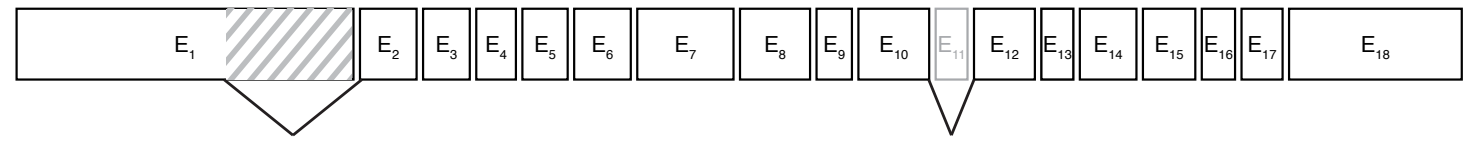

AC8E4

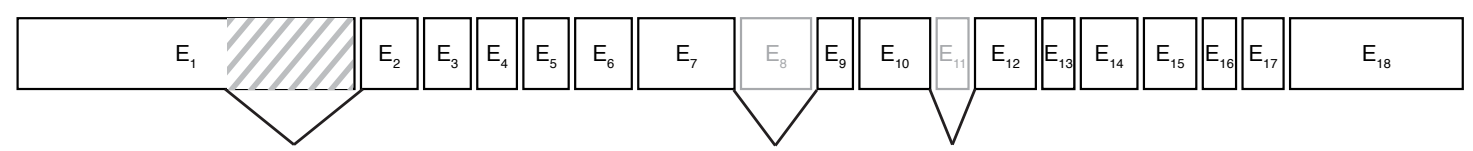

Figure S1 - Exon organization of AC8E variants

AC8E1-4 mRNAs all share a 414 bp deletion at the end of exon 1, corresponding to the light gray hatched box. Deletions of exon 8, exon 11 or both are represented by light gray boxes. 
A

B

AC8E1

AC8E2

AC8E4

AC8A

AC8E1

AC8E2
AC8E3

AC8E4

AC8A

AC8E1

AC8E2

AC8E3

AC8E4

AC8A

AC8E1

AC8E2

AC8E3

AC8E4

AC8A

AC8E1

AC8E 3

AC8E4

AC8A

AC8E1

AC8E2

AC8A

AC8E1

AC8E2

AC8E

AC8A

C8E1

AC8E2

AC8E3

AC8A

AC8E1

AC8E2

AC8E3

AC8E4

AC8A

AC8E1

AC8E2
AC8E3

AC8E4

AC8A

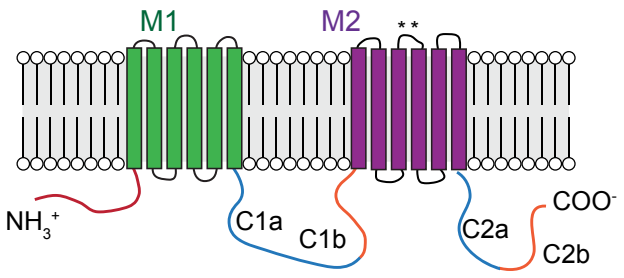

MELSDVHCLSGSEELYTIHPTPPAADGGSGSRPORLLWQTAVRHITEORFIHGHRGGGGGGSRKASNPAGSGPNHHAPOLSSDSVLPLYSLGSGERAHNTGGTKVFPERSGSGSASGSGGGGDLG ELLSVHCLSGSEELYTI HPTPPAADGGSGSRPQRLLWQTAVRHITEQRF I HGHRGGGGGSRKASNPAGSGPNHHAPQLSSDSVLPLYSLGSGERAHNTGGTKVFPERSGSGSASGSGGGGDLC LLSDVHCLSGSEELYTI IPTPPAADGGSGSRPQRLLWQTAVRHITEQRF I HGHRGGGGGGSRKASNPAGSGPNHHAPQLSSDSVLPLYSLGSGERAHNTGGTKVFPERSGSGSASGSGGGGDLG MLSDVHCLSGSEELYTIHPTPPAADGGSGSRPQRLLWOTAVRHITEQRF I HGHRGGGGGGSRASNPAGSGPNHHAPOLSSDSVLPLYSLGSGERAHNTGGTKVFPERSGSGSASGSGGGGDL $\mathrm{N}$-terminal domain

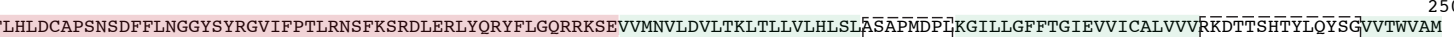
FLHLDCAPSNSDFFLNGGYSYRGVIFPTLRNSFKSRDLERLYQRYFLGQRRKSE--

LHLDCAPSNSDFFLNGGYSYRGVIFPTLRNSFKSRDLERLYQRYFLGQRRKSE-- -

LHLDCAPSNSDFFLNGGYSYRGVIFPTLRNSFKSRDLERLYQRYFLGQRRKSE---1-19.

LHLDCAPSNSDFFLNGGYSYRGVIFPTLRNSFKSRDLERLYQRYFLGQRRKS

TM1

I

EL1

TM2

IL1

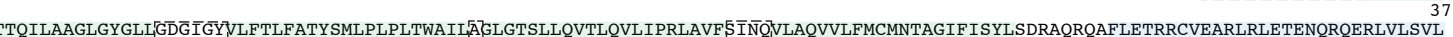

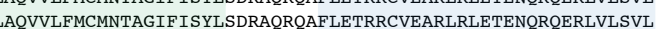

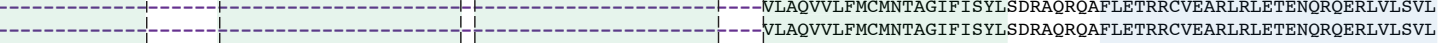

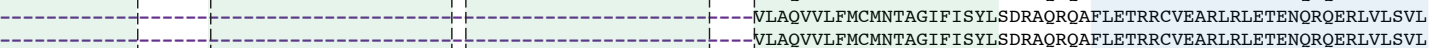

TM3

트는

TM4

IL2

Eㅡ는

TM6

C1a domain

PRFVVLEMINDMTNVEDEHLOHOFHRIYIHRYENVSILFADVKGFTNLSTTLSAOELVRMLNELFARFDRLAHEHHCLRIKILGDCYYCVSGLPEPRODHAHCCVEMGLSMIKTIRFVRSRTKHD RFVVLEMINDMTNVEDEHLOHOFHRIY IHRYENVSILFADVKGFTNLSTTLSAOELVRMLNELFARFDRLAHEHHCLRIKILGDCYYCVSGLPEPRODHAHCCVEMGLSMIKTIRFVRSRTKHD RFVVLEMINDMTNVEDEHLOHOFHRIY I HRYENVSILFADVKGFTNLSTTLSAOELVRMLNELFARFDRLAHEHHCLRIKILGDCYYCVSGLPEPRQDHAHCCVEMGLSMIKTIRFVRSRTKHD RFVVLEMINDMTNVEDEHLOHOFHRIY I HRYENVSILFADVKGFTNLSTTLSAOELVRMLNELFARFDRLAHEHHCLRIKILGDCYYCVSGLPEPRODHAHCCVEMGLSMIKTIRFVRSRTKHD C1a domain

VDMRIGIHSGSVLCGVLGIRKWOFDVWSWDVDIANKLESGGIPGRIHISKATLDCLSGDYNVEEGHGKERNEFLRKHNIETYLIKOPEESLISLPEDIVKESVSCSDRRNSGATETEGSWSPETP VDMRIGIHSGSVLCGVLGLRKWOFDVWSWDVDIANKLESGGIPGRIHISKATLDCLSGDYNVEEGHGKERNEFLRKHNIETYLIKOPEESLISLPEDIVKESVSCSDRRNSGATFTEGSWSPELP VDMRIGIHSGSVLCGVLGLRKWOFDVWSWDVDIANKLESGGIPGRIHISKATLDCLSGDYNVFEGHGKFRNEFLRKHNIETYLIKOPEESLLSLPEDIVKESVSCSDRRNSGATFTEGSWSPEI VDMRITIHSGSVICGULI RWOFDVWSWDVDIANKL DDRTII

C1a domain

C1b domain

FDNIVGKONTLAALTRNSINLLPNHLAOALHVOSGPEEINKRIEHTIDLRSGDKLRREHIKPFSLMFKDSSLEHKYSOMRDEVFKSNLVCAFIVLLFITAIOSLLPSSRTMPMTIOFSILTML 750 作

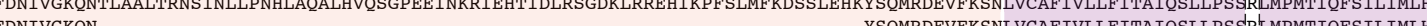

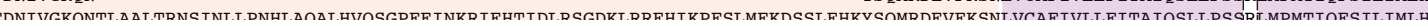
C1b domain TM7 EL4 TM8

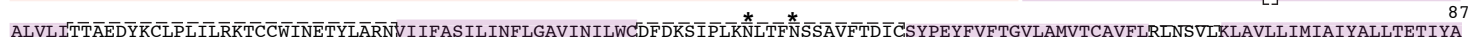
ALV 作 A A

IL $\underline{3}$ TM9

ELL5 FVFTGVL

ILL_ I TM11

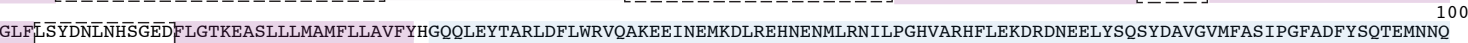
GLFLSYDNLNHSGEDFLGTKEASLLLMAMFLLAVFYHGOOLFYTARLDFLWRVOAKEEINEMKDLREHNENMLRNILPGHVARHFLEKDRDNEELYSOSYDAVGVMFAS IPGFADFYSOTEMNNO

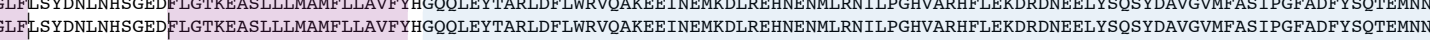
GLFLSYDNLNHSGEDFLGTKEASLLLMAMFLLAVFYHGQOLEYTARLDFLWRVQAKEE INEMKDLREHNENMLRNILPGHVARHFLEKDRDNEELYSOSYDAVGVMFASIPGFADFY SOTEMNN GLFL SYDNLNHSGEDFLGTKEASLLLMAMFLLAVFYHGOQLEYTARLDFLWRVQAKEE INEMKDLREHNENMLRNILPGHVARHFLEKDRDNEELYSOSYDAVGVMFASIPGFADFYSOTEMNN L_EL TM12 C2a domain

GVECLRLLNEIIADFDELLGEDRFODIEKIKTIGSTYMAVSGLSPEKOOCEDKWGHLCALADFSLALTESIOEINKHSFNNFELRIGISHGSVVAGVIGAKKPOYDIWGKTVNLASRMDSTGVSG 112 GVECLRLLNEI IADFDELLGEDRFQDIEKIKTIGSTYMAVSGLSPEKQQCEDKWGHLCALADF SLALTESIQE INKHSFNNFELRIGISHGSVVAGVIGAKKPQYDIWGKTVNLASRMDSTGVSS GVECLRLLNEIIADF DELLGEDRFODIEKIKTIGSTYMAVSGLSPEKOOCEDKWGHLCALADF SLALTESIOEINKHSFNNFELRIGISHGSVVAGVIGAKKPOYDIWGKTVNLASRMDSTGVSG GVECLRLLNEIIADFDELLGEDRFODIEKIKTIGSTMAVSGLSPEKOOCEDKWGHLCALADF SLALTES IOE INKHSFNNFELRIGISHGSVVAGVIGAKKPOYDIWGKTVNLASRMDSTGVSG GVECLRLLNEI IADFDELLGEDRFODIEKI KTIGSTYMAVSGLSPEKOOCEDKWGHLCALADF SLALTESIOEINKHSFNNFELRIGISHGSVVAGVIGAKKPOYDIWGKTVNLASRMDSTGVSG C2a domain

RIQVPEETYLILKDOGFAFDYRGEIYVKGISEOEGKI KTYFLLGRVOPNPF ILPPRRLPGQYSLAAVVLGLVOSLNROROKOLLNENSNSGI IKSHYNRRTLLTPSGPEPGAQAEGTDKSDLP 1248 RIOVPEETYLILKDOGFAFDYRGE I YVKGISEOEGKI KTYFLLGRVOPNPF ILPPRRLPGQYSLAAVVLGLVOSLNROROKOLLNENSNSGI IKSHYNRRTLLTPSGPEPGAQAEGTDKSDLP RIQVPEETYLILKDOGFAFDYRGEIYVKGISEQEGKIKTYFLLGRVQPNPF ILPPRRLPGQYSLAAVVLGLVQSLNRQRQKQLLNENSNSGI IKSHYNRRTLLTPSGPEPGAQAEGTDKSDLP RIQVPEETYLILKDQGFAFDYRGEIYVKGISEOEGKIKTYFLLGRVQPNPF ILPPRRLPGQYSLAAVVLGLVOSLNRQROKOLLNENSNSGI IKSHYNRRTLLTPSGPEPGAQAEGTDKSDLP RIOVPEETYLILKDOGFAFDYRGEI YVKGISEQEGKIKTYFLLGRVQPNPF ILPPRRLPGOYSLAAVVLGLVOSLNROROKOLLNENSNSGI IKSHYNRRTLLTPSGPEPGAQAEGTDKSDLP

C2b domain

Figure S2 - Multiple amino acid sequence alignment and structural domains of the AC8A and AC8E1-4 proteins

A Schematic representation of AC8A. 
B Amino acid sequence alignment of Rattus norvegicus AC8A, AC8E1, AC8E2, AC8E3 and AC8E4. Structural domains are indicated beneath the sequences. The missing amino acids in each AC8E isoform are represented by dotted lines.

In (A) and (B), the N-terminal domain is shown in red. M1 and M2 are the two transmembrane clusters composed of the membrane-spanning domains TM1-6 (green) and TM7-12 (purple), respectively. Membrane-spanning domains are linked by intracellular (IL) or extracellular (EL) loops. The $\mathrm{C} 1$ domain is subdivided into $\mathrm{Cla}$ (blue) and $\mathrm{C} 1 \mathrm{~b}$ (orange) domains. The $\mathrm{C} 2$ region is also subdivided in two regions, $\mathrm{C} 2 \mathrm{a}$ (blue) and $\mathrm{C} 2 \mathrm{~b}$ (orange). The $\mathrm{C} 1 \mathrm{a}$ and $\mathrm{C} 2 \mathrm{a}$ regions (blue) form the catalytic core. M2 presents two $\mathrm{N}$-glycosylation sites in extracellular loop 5 , indicated by *.

A

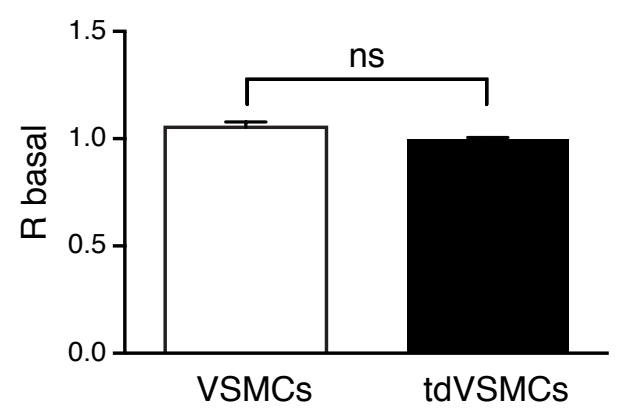

B

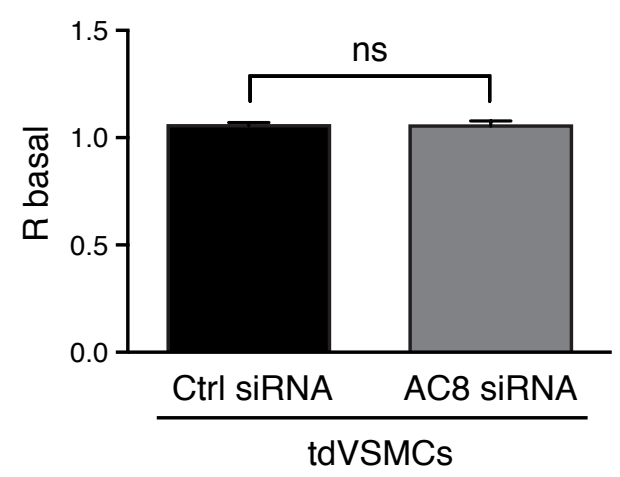

\section{Figure S3 - Basal [cAMP $]_{i}$ in VSMCs}

A, B Baseline ratio ( $\mathrm{R}$ basal) in contractile VSMCs and tdVSMCs (A) and in tdVSMCs transfected with control (Ctrl) or AC8 siRNA (B). Bars indicate the mean $\mathrm{R}$ basal $\pm \mathrm{SEM}$ of at least $N=4$ independent experiments performed on $n=10$ to 20 individual cells. In (A), R basal values were as follows: for contractile VSMCs: $1.05 \pm 0.02, N=5$; for tdVSMCs: 1.00 $\pm 0.01, N=4, P=0.1905$. In (B), R basal values were as follows: for Ctrl siRNA-transfected tdVSMCs: $1.06 \pm 0.01, N=10$; for AC8 siRNA-transfected tdVSMCs: $1.05 \pm 0.02, N=10, P$ $=0.5191$. Side-by-side comparisons were performed with the nonparametric Mann-Whitney test for unpaired data. ns: not significant. 
A

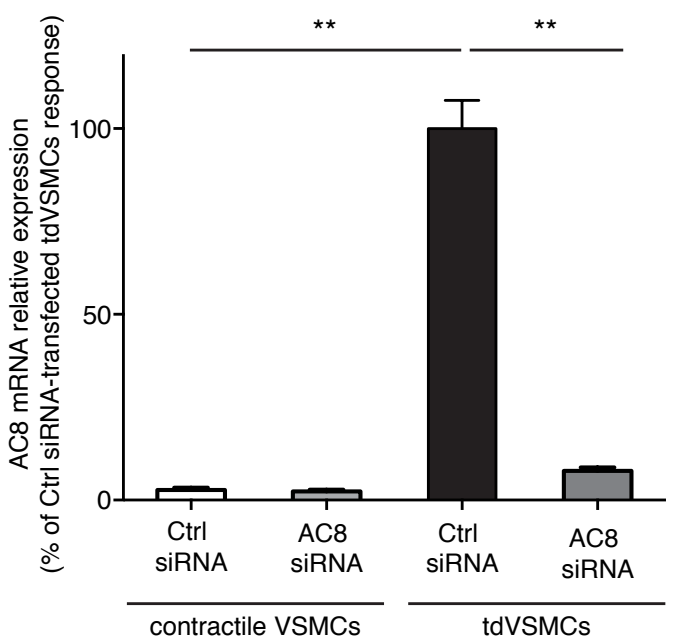

B

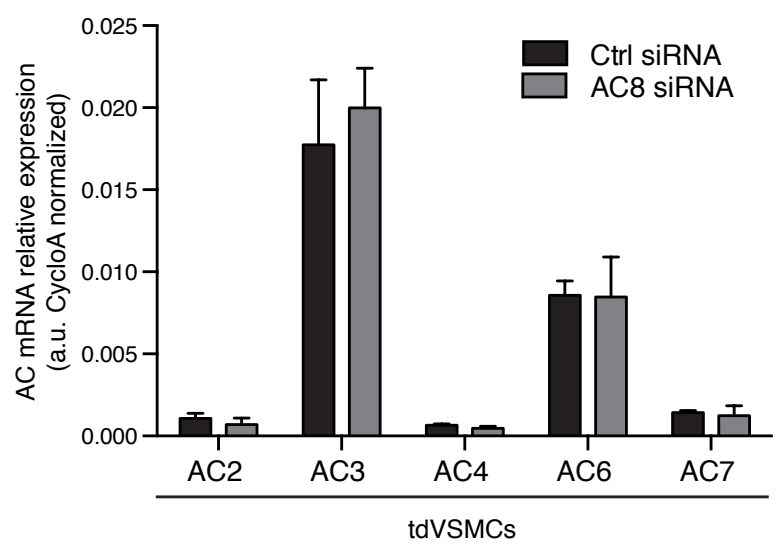

\section{Figure S4 - Efficiency and specificity of AC8 siRNA}

A Relative level of AC8 mRNAs in contractile or transdifferentiated (td) VSMCs transfected with control or AC8 siRNA. Results are expressed as percentages relative to tdVSMCs transfected with control siRNA and are normalized relative to cyclophilin A (cycloA) mRNA levels. Bars represent the means \pm SEM of 4 independent experiments. Sideby-side comparisons were performed with the nonparametric Mann-Whitney test for unpaired data. **: $P<0.01$.

B ACs mRNA expression in tdVSMCs transfected with control (Ctrl) or AC8 siRNA. Results are expressed in arbitrary units (a.u.), normalized relative to cyclophilin A (cycloA) mRNA expression. Bars represent the means \pm SEM of 3 independent experiments. 


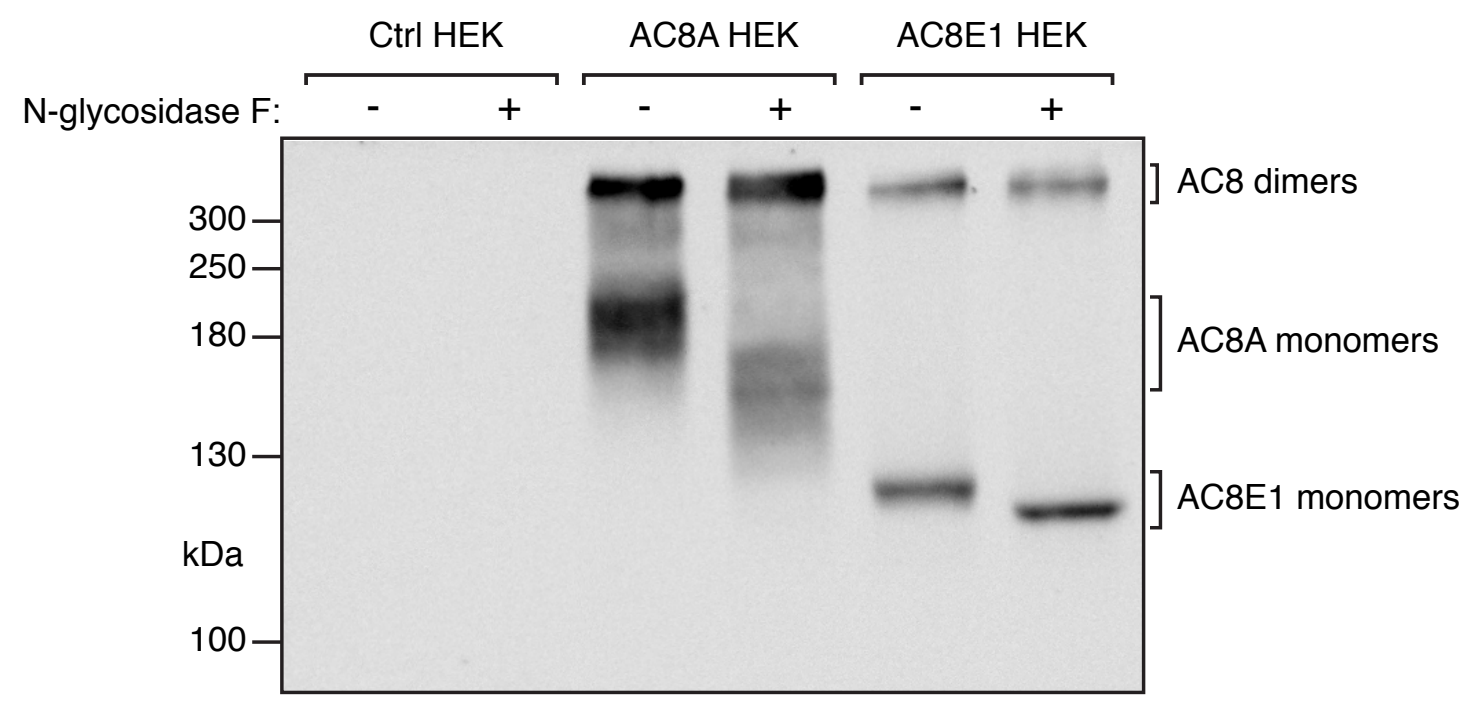

\section{Figure S5 - AC8E1 exists as N-glycosylated species}

Western blot of total extracts issued from HEK-293 cells stably expressing AC8A or AC8E1, treated with $(+)$ or without (-) N-glycosidase F (500 units). The western blot shown is representative of 3 independent experiments. 


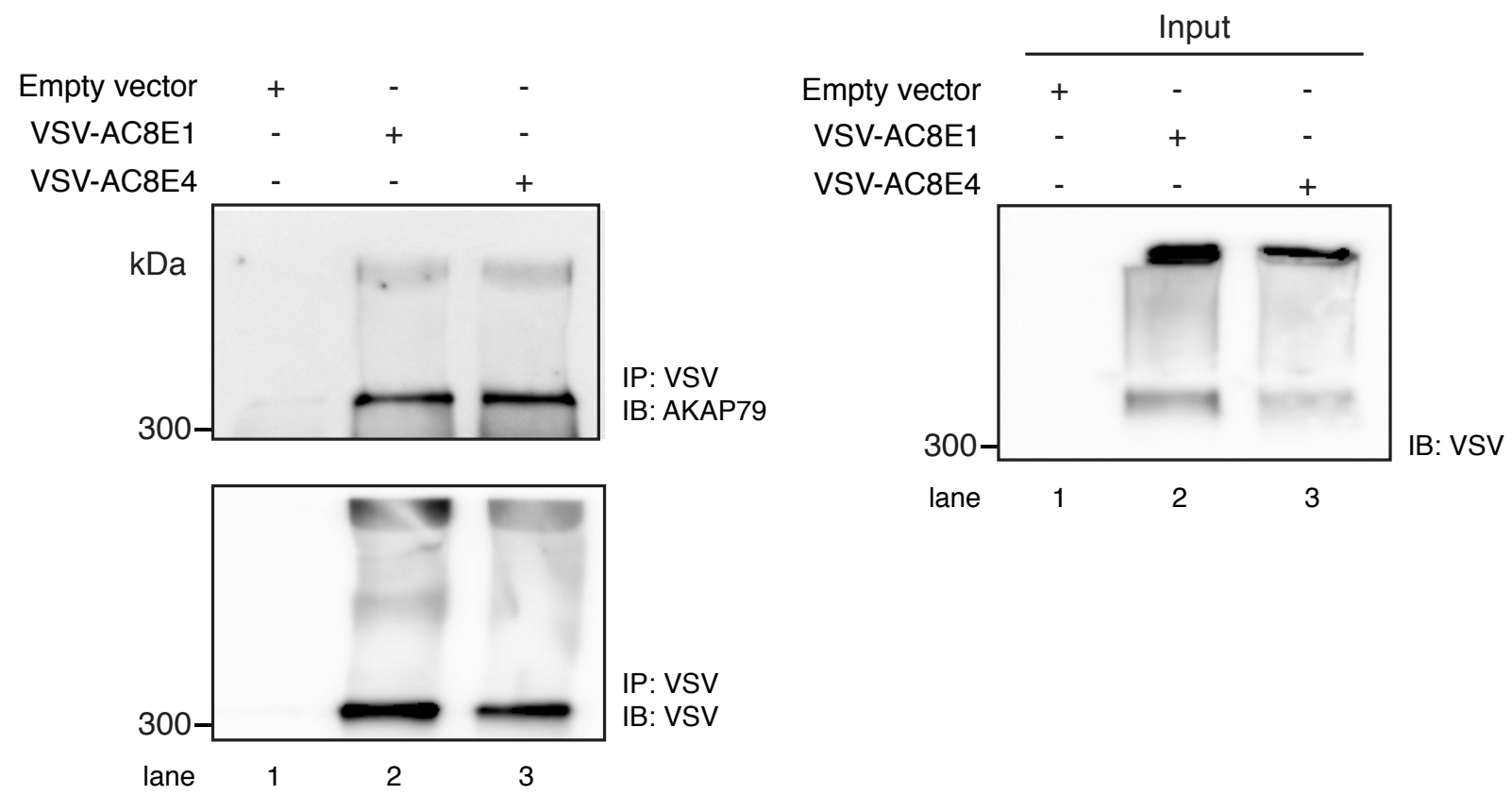

\section{Figure S6 - AC8E1 and AC8E4 interact with AKAP79}

HEK-293 cells were transiently transfected with empty vector (pcDNA3-VSV) or plasmid encoding VSV-AC8E1 or VSV-AC8E4. Left panel: western blots showing coimmunoprecipitation of AKAP79 with AC8E1 and AC8E4. Immunoprecipitation (IP) was performed with anti-VSV antibody and immunoblots (IBs) with anti-AKAP79 or anti-VSV antibodies. Right panel: western blot showing AC8E1 and AC8E4 levels in crude cell lysates performed with anti-VSV antibody. The western blots shown are representative of 3 independent experiments. 
Supplementary tables

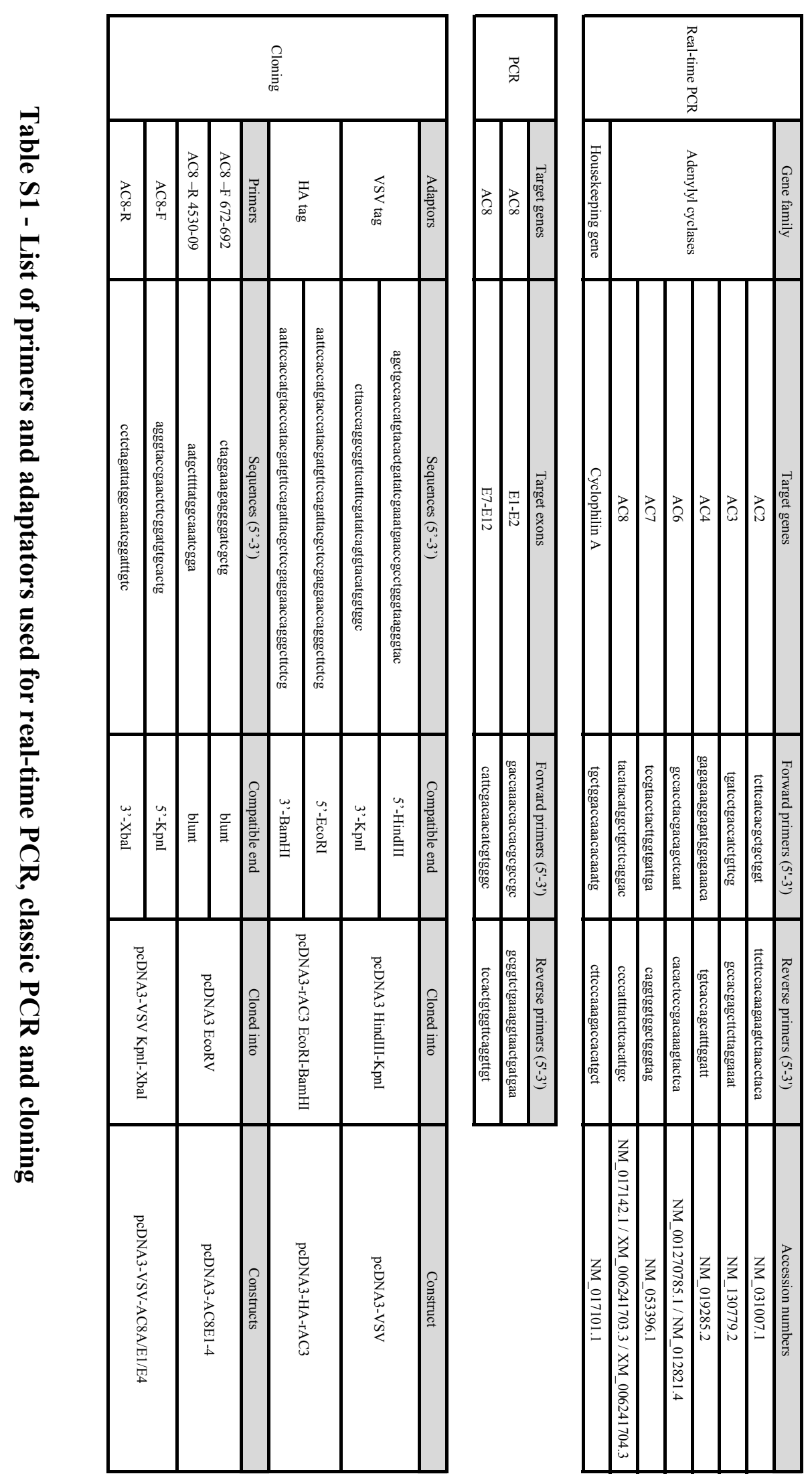




\begin{tabular}{|c|c|c|c|c|}
\hline Target proteins & Sources & Applications & Manufacturers & Catalog numbers \\
\hline AC8 (C-terminus) & goat polyclonal & IB, IF & Santa Cruz Biotechnology, Inc. & sc-1967 \\
\hline AC3 (C-terminus) & rabbit polyclonal & IB & Santa Cruz Biotechnology, Inc. & sc-588 \\
\hline GAPDH & rabbit monoclonal & IB & Abcam & Ab181602 \\
\hline$\beta$-actin & mouse monoclonal [AC-15] & IB & Sigma-Aldrich & A5441 \\
\hline$\beta 1$-integrin & rabbit monoclonal [EP1041Y] & IB & Millipore & $50-171-223$ \\
\hline Calnexin & rabbit polyclonal & IB & Santa Cruz Biotechnology, Inc. & sc-11397 \\
\hline COX IV & goat polyclonal & IB & Santa Cruz Biotechnology, Inc. & sc-69359 \\
\hline AKAP79 & mouse monoclonal & IB & BD Biosciences & 610314 \\
\hline \multirow{2}{*}{ VSV tag } & mouse monoclonal [P5D4] & IB & Abcam & ab50549 \\
\cline { 2 - 5 } & goat polyclonal & IP, IF & Abcam & ab3861 \\
\hline HA tag & mouse monoclonal [16B12] & IP, IF & Abcam & ab130275 \\
\hline
\end{tabular}

Table S2 - List of antibodies used in immunoblots, immunoprecipitation and immunofluorescence experiments 